\title{
Heuristic techniques for modelling machine spinning processes
}

\author{
Roman Stryczek $^{1}$ (1) Kamil Wyrobek ${ }^{1}$ (]) \\ Received: 7 November 2019 / Accepted: 25 September 2020 / Published online: 15 October 2020 \\ (c) The Author(s) 2020
}

\begin{abstract}
In spite of many efforts made a complete model of machine spinning processes, due to its complexity, multidimensionality of the decision space and the present state of knowledge, is unachievable. The paper addresses the issues of constructing a local process model to enable the search for a locally optimal course of the process, within a short time and with the cost as low as possible. Comparison was made between the theoretically well-grounded response surface designs method with a few approaches to the model construction based on intuitively understood heuristic bases justified by their successful practical applications. In order to determine a set of Pareto-optimal solutions for a discrete decision space, the durations of process execution were generated through a virtual simulation. In order to outline and justify the adopted solutions a comprehensive example of the practical construction of the machine spinning process model was presented, including its various versions. The results obtained were validated and evaluated. The main utilitarian conclusion is the indication whereby basing on a partial experiment plan it is possible, thanks to simple heuristic methods, to obtain Pareto-optimal solutions which are close to those obtained when the full experiment plan is carried out.
\end{abstract}

Keywords Machine spinning $\cdot$ Response surface designs $\cdot$ Case based reasoning $\cdot$ Potential function method $\cdot$ Madaline

\section{Introduction}

The constantly developing field of science, known as knowledge engineering, facilitates the acquisition, structuring, storage and processing of the manufacturing knowledge for engineers. One of the techniques readily deployed in knowledge engineering is the construction of models describing knowledge in a given field, their validation and searching for optimal solutions based thereon. The demand for intelligent planning of manufacturing processes rises as a reflection of the highly competitive market environment that requires, lower production costs shortening production cycle and providing more stable process planning ability (Ma et al. 2020; Ye et al. 2020). A virtual model, although simplified, may provide a range of valuable cognitive information, contributing to the understanding and, consequently, development of a given manufacturing technique. Modelling allows for check-

Kamil Wyrobek

kwyrobek@ath.bielsko.pl

Roman Stryczek

rstryczek@ath.bielsko.pl

1 Faculty of Mechanical Engineering and Computer Science, University of Bielsko-Biala, Bielsko-Biala, Poland ing design assumptions and possibly their quick verification at a relatively low cost.

The machining processes carried out on tool machines involve the interaction of the tool and the workpiece. Including all the interactions between the tool and the workpiece in the design process, as well as the dynamic behaviour of the machine tool on which the process will be carried out, allow for increasingly more faithful models of the production process, with an increasing level of integration. Process models allow for designing production processes in the off-line mode, contribute to the systematisation, transfer and dissemination of knowledge in a given field, while facilitating the study of processes, improvement of performance and quality indicators of these processes, reduction of undesirable production downtime, as well as rational selection of tools and machine tools. The work (Kleiner et al. 2002), presents a statement, gaining importance in the context of this study, whereby the most effective way to capture defects in a product is a classification expressed by means of categorising models.

Manufacturing operations are tested experimentally, analytically and numerically. Experimental methods are both expensive and time consuming (Gok 2015b). Generally, there are two different approaches to generating a knowl- 
edge model for a given manufacturing process. The first one is based on the deployment of advanced IT tools, creating an extensive mathematical modelling environment, allowing one to describe all the variable model parameters with relative accuracy and relationships between them, and run the simulation process. The second simplified approach is to build a knowledge model based on artificial intelligence methods, which include heuristic solutions based on incomplete and uncertain knowledge but providing rational solutions. Among the precision methods, the finite element method (FEM) has been the most popular in the last two decades. An example of a successful FEM three-dimensional application for the selection of turning parameters is the work (Gok 2015b). The comparison of the temperature, main cutting force and pressure obtained from experimental and numerical analyses was performed. The author draws attention to the importance of including appropriate considerations for loading and boundary conditions in the model being developed. The possibilities of using FEM to study the milling process are presented in (Gok et al. 2017). The current state and conditions of FEM in the context of metal spinning are presented in doctoral dissertations (Wang 2012; Rentsch 2018). However, the calculation time for the full metal spinning process turned out to be too long, not only for practical application, but also for theoretical studies. Most of the works was limited to the simulation of the first run of the forming roller, but as noted in (Hayama and Murota 1963), deformation modes are different at the beginning and end of the process, hence the practical usefulness of this type of models is insignificant. It should be borne in mind that FEM requires very high qualifications from the user, being able to formulate the boundary conditions of the method and correctly interpret the results of processing. Of course, the development of FEM software plus the increase in the computing power of the future generations of computers will make FEM attractive.

The reference method to which the heuristic methods used in this work were compared was the popular response surface design (RSD) method, introduced in 1951 by Box and Wilson, also known as response surface analysis (RSA) or response surface methodology (RSM). It is a methodology utilising a combination of statistical and mathematical techniques to design and optimise processes using a first or second order polynomial model. An example of using this method is contained in (Gok 2013), where it was used to build a model of the influence exerted by the cutting parameters and different styles of the tool path on the cutting force and tool deflection during ball end milling. In another work (Gok 2015a), the purpose of the research was to determine a mathematical model of the dependence of average surface roughness, maximum roughness as well as the main cutting force and feed force on the parameters of turning during this operation using RSA. The adequacy of the developed mathematical model was confirmed with the ANOVA method.
A large variety of heuristic approaches used to build the knowledge model and then optimise manufacturing processes was characterised, among others, in the works: Mukherjee and Kumar (2006), Stryczek (2007), Mao et al. (2010), Li et al. (2018). Searching for solutions through analogy is an attractive alternative in a situation where carrying out a full knowledge acquisition process is difficult, expensive, time consuming or even impossible. The case based reasoning (CBR) method has already proved its value in many professional applications. This approach is closely related to human reasoning and is based on the re-use of proven solutions and stored case information in order to solve new problems. An approach based on the CBR method for metal spinning was proposed by Ewers (2005). The author compared the adaptive sequential optimization procedure (ASOP) procedure to the popular one-factor-at-a-time (OFAT) method, stating that ASOP improves the process by approximately $37 \%$. However, the ASOP method requires a large case database. Khosravani and Nasiri (2020) review CBR systems that are used in injection molding for different purposes, such as process design, processing parameters, fault diagnose, and enhancement of quality control. In addition, they discuss trends for utilization of CBR in different phases of injection molding. In another work (Göbel et al. 2005), at the first stage of the design sequence, the prediction of initial parameter settings was based on the analysis of previous cases. The first adaptation of the pre-selected parameters is then carried out on the fuzzy model. In the next step, model-based optimisation is performed using a statistical design of experiments. The CBR method determines whether a given case belongs to a specific category on the basis of the similarity to the nearest neighbouring case in the adopted metric. It does not take into account the impact of several adjacent cases, possibly belonging to different categories. The potential function method is not burdened with this disadvantage. The potential functions method (PFM) belongs to the classic, intuitive methods of image recognition (Aizerman et al. 1964). It refers to a similar function, performed by potential in physics, determined for any point in space, and depending on the point of location of the source of potential. The last method of building a local model of the metal spinning process, analysed in this work, is neural networks. However, following the principle whereby the method should be not only useful but also intuitively understood by the direct user, one of the simplest networks known since the 1960s under the name Madaline (Widrow and Lehr 1990) was selected.

A comprehensive review of the methods used to optimise the processes implemented on machine tools is presented in (Mukherjee and Kumar 2006). Some important modelling and optimization techniques presented Vankata Rao (2011). The current set of works dealing with the multi- 
criterion optimisation of machining parameters can be found in (Vergara-Villegas et al. 2018).

\section{Premises and barriers for the application of machine spinning}

Machine spinning processes are a version of plastic processing where through turning a blank in the form of a circle made of metal with appropriate plastic properties one can obtain thin-walled elements relatively quickly and inexpensively. These processes can be divided into manual and machine ones. Manual spinning has traditions dating back to the ancient Egypt though it has remained popular to this day. A worker with appropriate experience guides the tool manually imparting the desired shape to the workpiece. The process requires a permanent presence of a high-qualified worker and their constant effort requiring considerable energy. Along with the development of CNC machine tools machine spinning is becoming increasingly popular allowing this process to be performed with full automation. This process can be executed on specially adapted spinning machines equipped with a tailstock which improves process stability. An alternative for that type of machine tools is provided by popular, standard CNC lathes with which a limited but still extensive range of products may be shaped using the spinning method. The three components of tooling essential for the execution of the spinning process are: a forming roller, lathe mandrel, and a tailstock fastening the circle being spun (Fig. 1).

The premises for the application of standard CNC lathes for the execution of the spinning process are:

- availability of CNC lathes,

- no requirements for the presence of an employee with broad experience in spinning processes,

- possibility of the full automation of the production process,

- possibility to quickly change process parameters (process parameterisation), including the tool path,

- possibility to use flexible user cycles

- possibility to manufacture consumable tooling elements on the same machine tool,

- natural possibility of supplementing the process with additional machining treatments and the general advantages of the spinning process (Music et al. 2010), such as:

- material savings,

- energy savings,

- possibility of running short production series in economic conditions,

- simple, cheap, easily available and largely universal tooling,

- short idle time for production set-up,

- short time and low cost of launching the production of new products,
- possibility to obtain diverse, thin-walled parts,

- possibility to obtain a high-quality surface finish,

- improvement in strength properties of the material being formed.

The barriers of the machine spinning process include:

- high number of the factors affecting the correctness of the spinning process course,

- possibility of the occurrence of production defects (cracking/breaking, folding) at incorrect process parameters,

- no transfer of the knowledge on the effective tool paths of the forming roller movement and process parameters, complex mechanics of the spinning process, etc. (Music et al. 2010). Forecasting the evolution of stresses, deformations, and damage during the spinning process is particularly difficult,

- no machining cycles offered by the producers of standard numerical control systems for machine spinning. Development of a programme to control machine spinning process from scratch is a considerable challenge.

- difficulties in including considerations for an intricate shape of the working surface of the forming roller (Stryczek and Wyrobek 2017),

- comprehensive models of spinning process that would allow for simulation off-line design are not available.

This paper aims to mitigate the above barriers through the presentation and comparison of a few approaches to the construction of the local models of the spinning process. A manufacturer starting a spinning process but devoid of the sufficient knowledge on its course performs some tests which may result in production rejects. The use of optimization techniques presented in this article should allow for locating the local region of the spinning process stability quickly, limiting the number of test trials, minimizing the number of rejects and shortening the process of estimating the parameters of the spinning process which are close to the optimal ones.

\section{Modelling of machine spinning process}

In spite of a considerable number of works conducted since 1950s and striving to build a model of the machine spinning process this goal has not been achieved so far. There are many reasons for this situation and the most important ones lie in the high number of variable parameters determining the course of the process (Fig. 2), the complexity of the process and the lack of full knowledge on the mechanics of the machine spinning process. In the studies of spinning carried out so far certain experimental techniques were used to examine the mechanism of deformations and their evolution, mechanisms of the emergence of folds, formation of 
Fig. 1 View of the research stand for machine spinning based on a standard $\mathrm{CNC}$ lathe

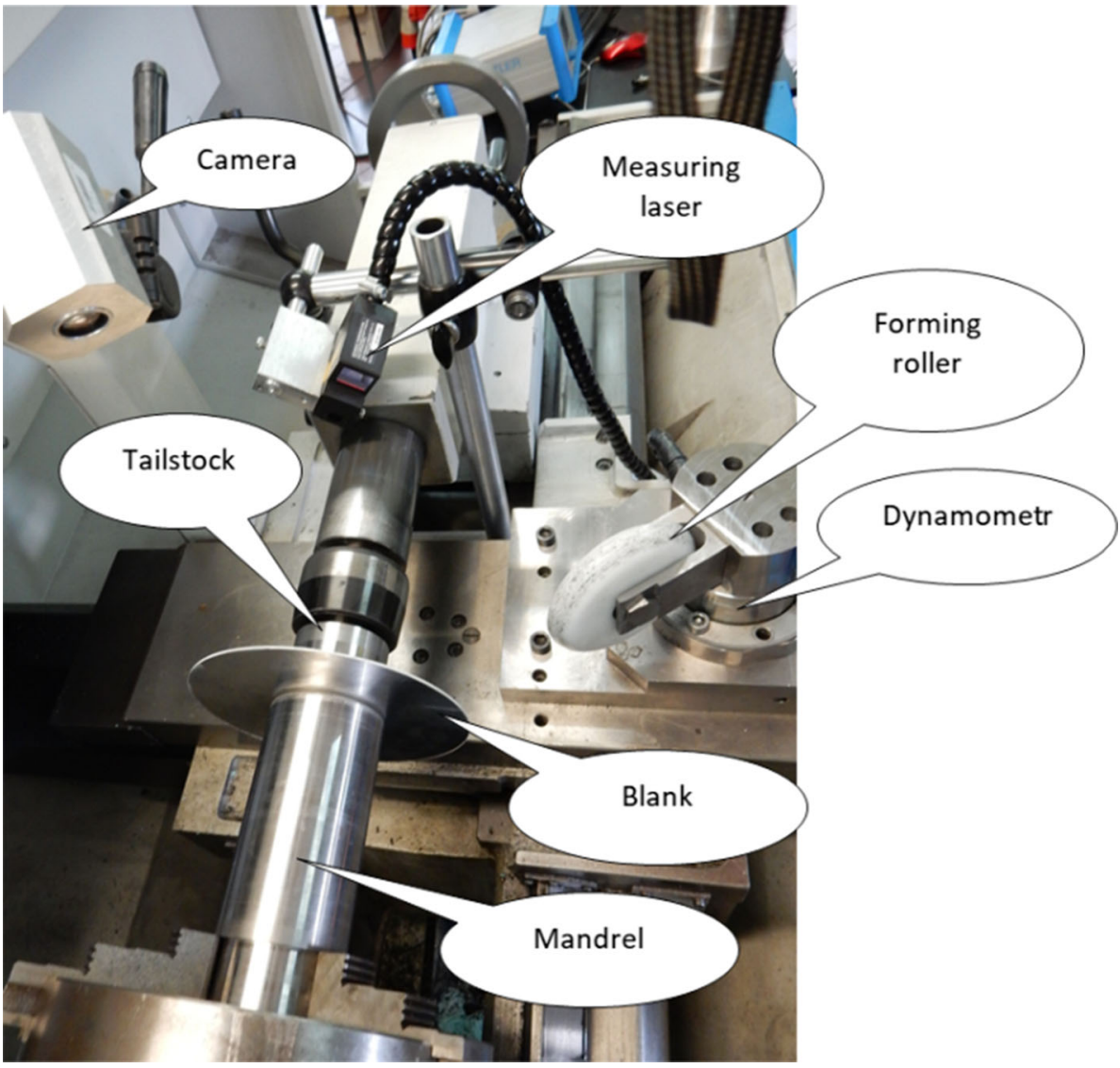

forces, surface quality, and optimization of product geometry. In theoretical techniques, analytic and numerical approaches were applied, a broad overview of which is contained in the study (Music et al. 2010). As regards earlier overview studies (Slater 1979) is worth mentioning, in which the author examines both the experimental and analytical models of spinning used to forecast the forming and folding forces, rim formation and surface finish. Forecasting the forces in the spinning process is useful in the design of spinning machines and tooling but it does not explain the occurrence of manufacturing defects where the analysis of stresses and deformation is necessary. In the paper (Auer et al. 2004) a review of various multifactorial methods of the reliable design of machine spinning process parameters is presented. A few variants were suggested and compared theoretically and empirically.

The development of a full model of spinning processes is not possible at present. One should focus on the construction of local models understood as those with a limited number (from 2 up to 4) of parameters, setting the other variables arbitrarily on a constant level which results from the acquired knowledge.

In workshop practice most of the values of input parameters are determined by the material stipulated by the design, required shape and product dimensions or available workshop tooling and equipment. In order to start the production of a new article the manufacturer selects only a few basic values determining the correctness of the process course and the quality of the articles being produced. The most essential one of them is the trajectory or tool path of the forming roller movement in successive passes and the roller feed.

The configuration of appropriate $\mathrm{CNC}$ programs can be recognised as the key aspect of the modern spinning process (Auer et al. 2004). The full cycle of machine spinning requires from a few to up to a dozen or so complex working passes. Analysing the trajectory of the forming roller every time when the production of a new article starts would be downright tedious and would delay the launch of serial production and generate costs. Because of that in workshop practice one of the proven parameterized tool paths generated by a flexible machine cycle should be selected. An example of such a path is presented in Fig. 3. The useful machine cycle is characterized by a rather small number of variable parameters which still allows adjustment of the parameters vital from the point of view of production quality and output. Within the scope of the path geometry, when such articles as mugs, funnels, etc. are manufactured, the selection of optimal increase in the $A i$ angle and the length of the horizontal semi-axis $L t$ of the ellipse limiting successive passes remains. An excessively high value of the $A i$ angle is the reason for production defects and may create folds (Fig. 4c). However, decreasing 
Fig. 2 Input and output parameters of the machine spinning process
Input parameters

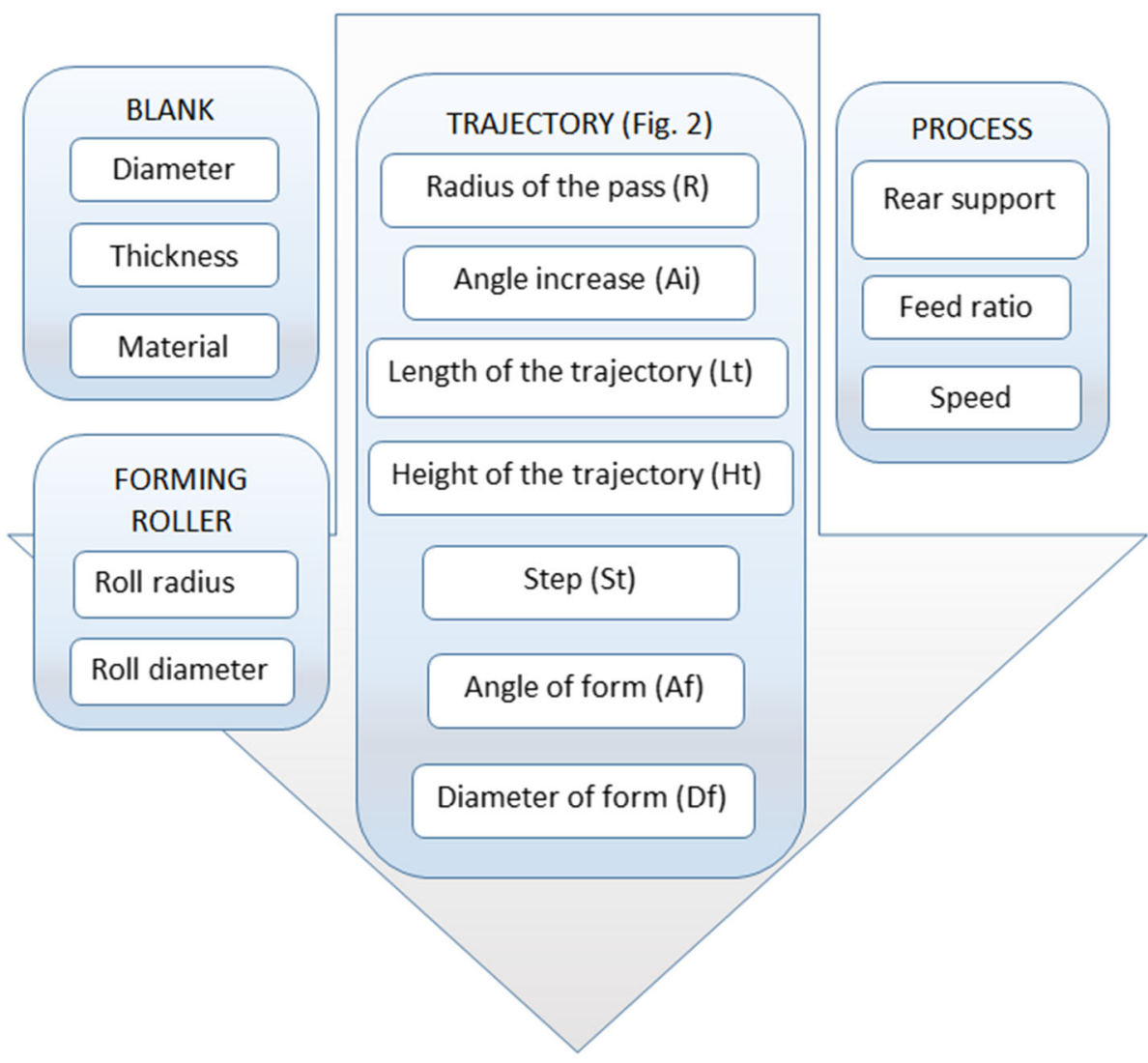

Output parameters

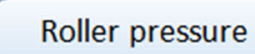

Cycle time the $A i$ angle causes the number of passes to increase, which obviously extends the cycle time. The $L t$ parameter affects the length of the piece being spun. An excessively low value of $L t$ leads to the creation of a flange and then thinning the wall and circumferential cracking (Fig. 4b). Higher $L t$ value extends the cycle time.

Another parameter generally affecting production quality and output is the working feed of the forming roller. It affects in an obvious way the machine cycle time. However, its impact on the creation of production defects is much more complex and most often requires test examinations.

In the first stage of the procedure optimizing the process, the region of stability should be established, such that defines a set of the points in the decision space enabling one to manufacture a correct product. The stability region, in the case
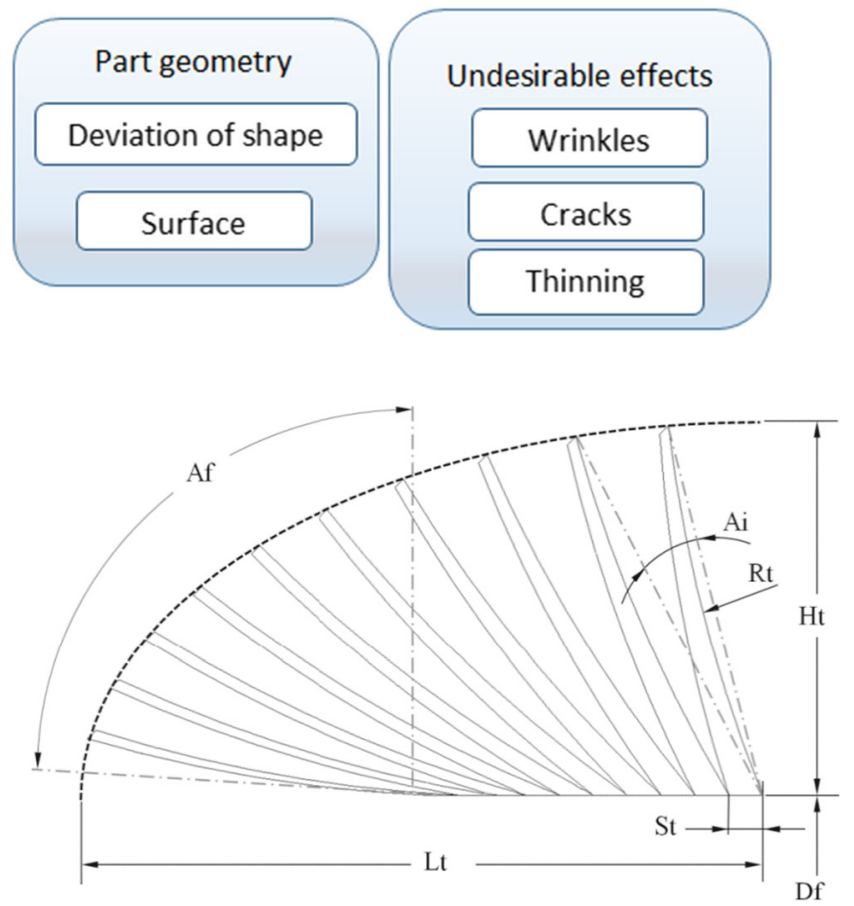

Fig. 3 Parameterized, curved concave trajectory of the forming roller in a machine spinning cycle 

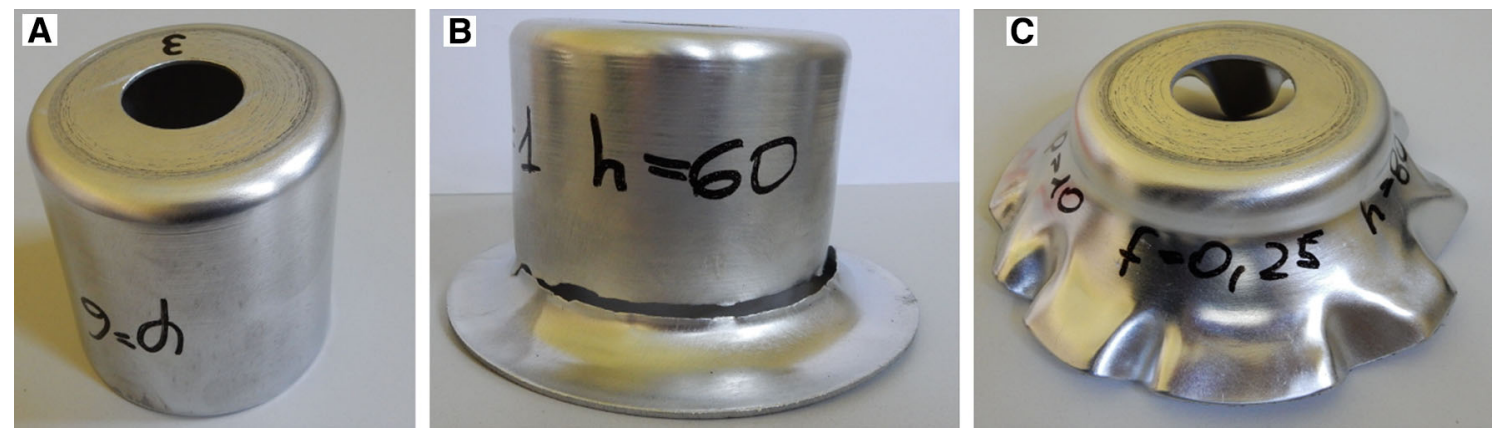

Fig. 4 a Correct product, b circumferential cracks, $\mathbf{c}$ folding

of machine spinning, has complex shapes in the multidimensional decision space. The points of that space, optimal from the point of view of the article and process parameters, are located on its narrowed edges and are adjacent to the forbidden area causing production defects (Ewers 2005; Göbel et al. 2005). The borders of those areas, being difficult to specify, should be considered fuzzy borders. Hence, to every point of the decision space the degree of affiliation with correct solutions that do not generate defects should be assigned. The optimisation of the machine spinning process should be considered as the task of determining the Pareto front in the criteria space which includes the degree of affiliation with solutions without defects and the process execution time.

Determining the duration of the execution of the machine spinning process on $\mathrm{CNC}$ machine tool off-line is not a problem at present, since the available virtual simulators of the control program course, based on the established machine cycle, may be applied. A master program with appropriate looping will automatically start the course of the machine cycle with the parameters corresponding to evenly distributed points in the decision space and will record the duration of the course in an appropriately formatted file, adapted for further processing. Figure 5 presents a scatter plot illustrating the distribution of the cycle course duration values in the decision space for a research example implemented in this study. The other variable parameters of the forming roller trajectory were constant: $\mathrm{Ht}=44 \mathrm{~mm}$, Rt $170 \mathrm{~mm}, \mathrm{Af}=85^{\circ}, \mathrm{St}$ $=5 \mathrm{~mm}$, Df $=\varphi 84 \mathrm{~mm}$. The radius of the forming roller was $15 \mathrm{~mm}$, and its outer diameter was $\varphi 110 \mathrm{~mm}$. The spindle revolutions were constant for all tests and amounted to 833 r.p.m. The dimensions of the semi-fished product: outer diameter $\varphi=170 \mathrm{~mm}$, thickness $=1.5 \mathrm{~mm}$, the metal sheet being spun-Al 99.5.

Having the set of the points constituting the Pareto front and the Pareto set corresponding to them in the decision space, the user selects the solution with satisfying time of execution of the machine cycle and the highest degree of affiliation with the solutions yielding no defects. In the opposite approach a subset of the solutions with a safe degree of affiliation with the solutions without defects can be defined

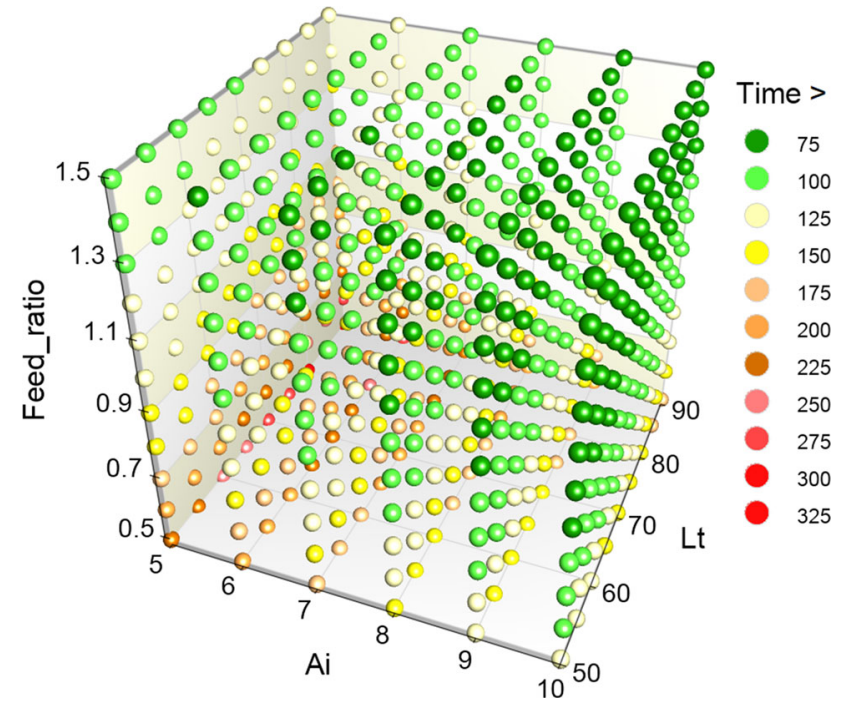

Fig. 5 The duration of the spinning cycle in the studied points of the decision space

and the solution with the shortest time of the execution of machine cycle can be found in this subset. This type of analysis can be still extended by the cost analysis in the context of the risk of the generation of losses.

Figure 6 shows the range of conducted experiments, modelling, and optimization of solutions. The starting point was 27 research experiments within the full, orthogonal research plan. Then a minimum plan was isolated according to the Taguchi orthogonal tables and the central composite plan of Face Centered CCF type. For those three plans procedures of calculating five different approaches were programmed and performed. Finally, optimization procedures were performed, and a set of Pareto-optimal solutions were established. For binary CBR and Madaline methods the optimization consisted only in the selection of the solution with the shortest cycle time, from among the solutions within the process stability region being estimated. 
Fig. 6 Considered modelling variants of the machine spinning process
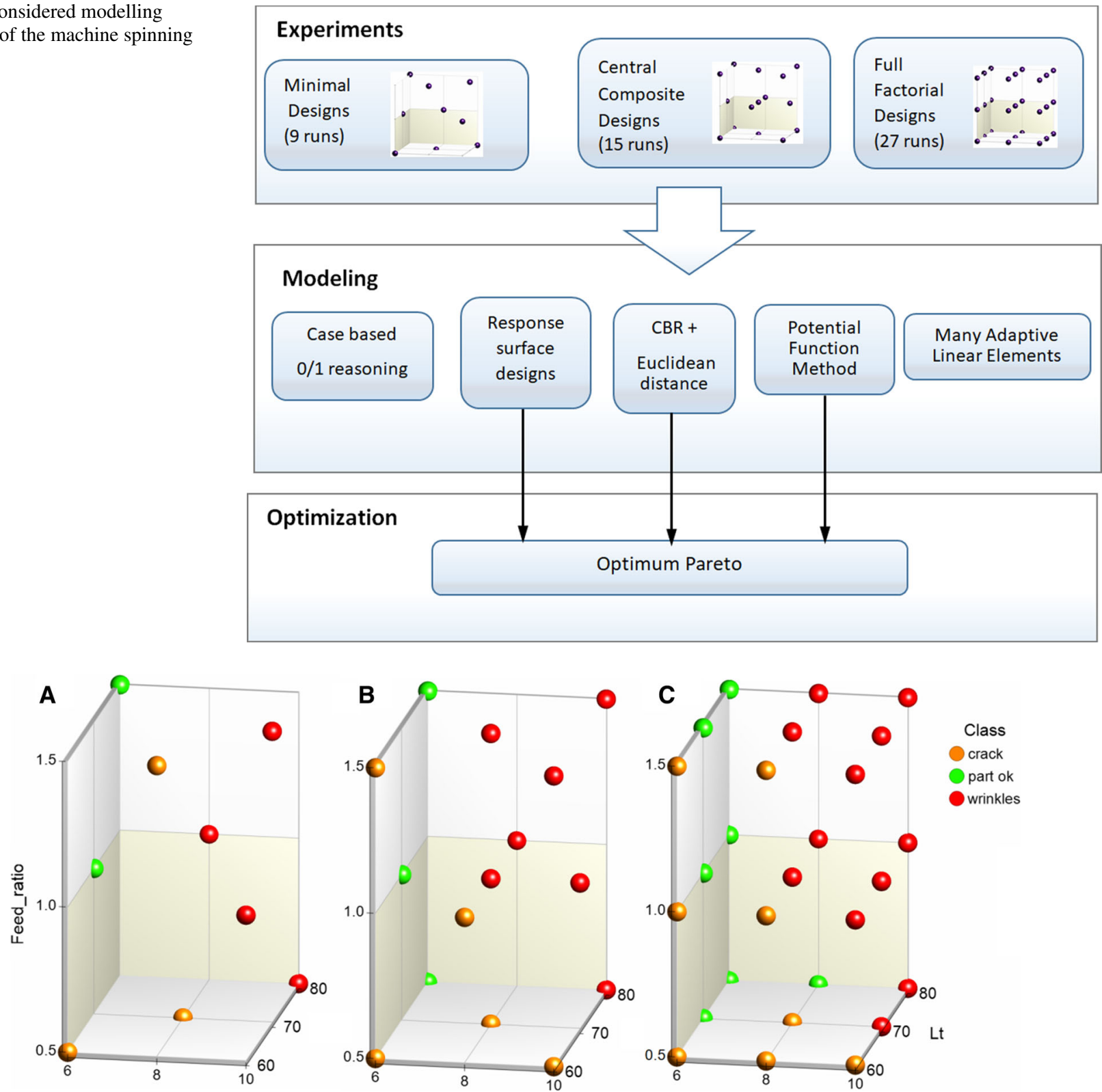

Fig. 7 The results of workshop tests for the minimal plan (a), central composite one (b), and full plan

\section{Performance of workshop experiments}

In Fig. 7 the results of workshop tests located in the decision space on the minimal plan (A), central composite one (B), and full plan (C). 3 classes have been visualised: part $\mathrm{OK}$ (green), occurrence of cracks and breaks due to the appearance of a rim on the circumference of the part (orange), and occurrence of folds (red). In subsequent part of the experiments a division into only two categories was applied: "part OK", and "part with defects". As it can be seen in the drawing the physical completion of the research plans involves the costs of production rejects: 7 for the minimal plan, 12 for the central composite one, and 20 for the full plan.

\section{Response surface design}

This method consists in the optimal selection of the parameters of the analytical model so that the proper responses of the Y surface will correspond as accurately as possible to the results obtained by experiments in the tested points of the decision space 1 for "part OK", and 0 for "part with defects". The response surfaces were built with the use of a polynomial of the 2nd degree which takes into account the impact of the values of process parameters and the interaction between them. Taking into account the general form of square model 

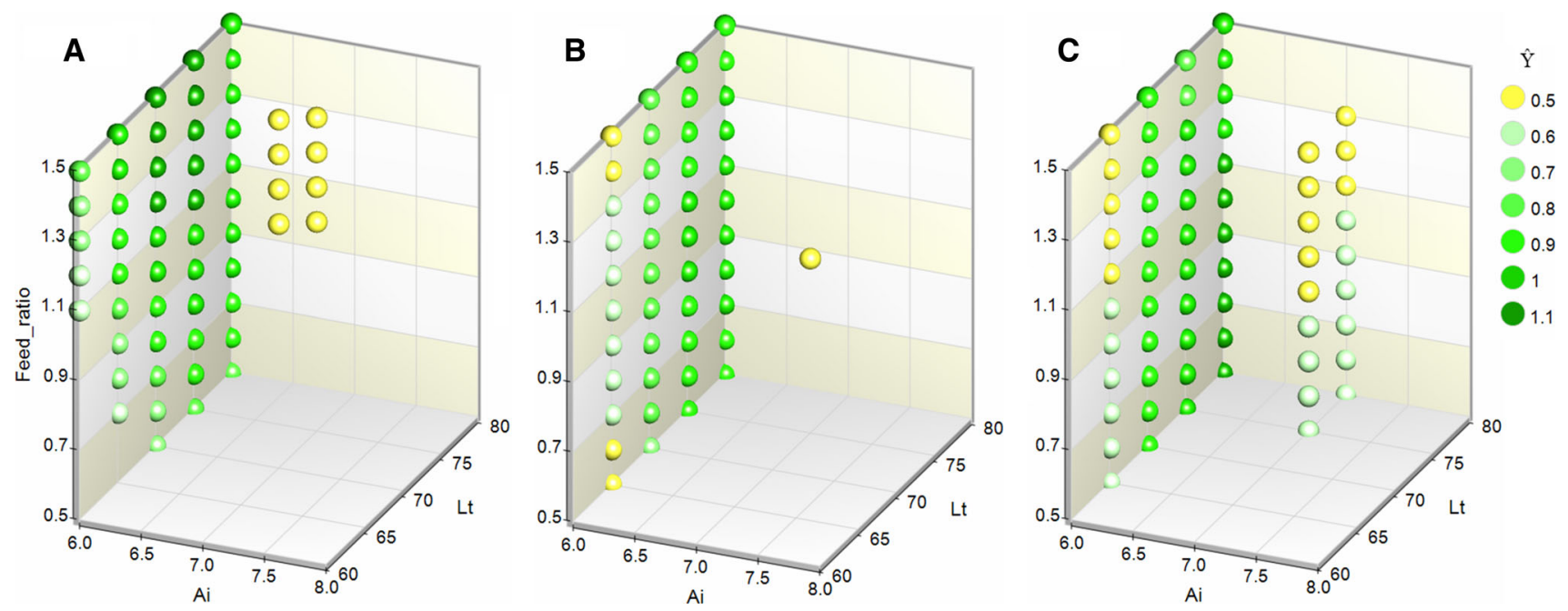

Fig. 8 The stability region determined by means of the RSD method basing on the minimal plan (a), and the central composite one (b)

which is given by the formula (1). The $\beta_{i}$ parameters have been optimized with the evolution algorithm.

$$
\begin{aligned}
\hat{\mathrm{Y}}= & \beta_{0}+\beta_{1} x_{1}+\beta_{2} x_{2}+\beta_{3} x_{3}+\beta_{4} x_{1} x_{2} \\
& +\beta_{5} x_{1} x_{3}+\beta_{6} x_{2} x_{3}+\beta_{11} x_{1}^{2}+\beta_{22} x_{2}^{2}+\beta_{33} x_{3}^{2}
\end{aligned}
$$

It was assumed that the decision space points forming the process stability region attain the response level higher or equal to 0.5 . The higher response level the higher is the affiliation with the stability region. In Fig. 8 the stability regions are presented successively for the minimal plan (A), central composite one (B) and the full plan (C). The area of the stability region is limited to the borders of the decision space determined by the limit values of the variable parameters taken into account in the experiments. The responses of the model for the points outside that space assume high absolute values that definitely exceed the assumed range for $\hat{Y}$ $[0,1]$. That is why their interpretation is burdened with high uncertainty.

\section{Drawing conclusions based on earlier cases}

The starting point in the CBR is the creation of a representative set of the cases whose solution is known. The next step is to find that case which is the most similar to the new problem in the case database. Finally, after verification, the new case is transferred to the case database whereby the knowledge in a given field is extended. In order to determine the similarity of individual cases characterised by the values of variable parameters expressed in different units, standardization of the decision space should be performed. For this purpose, in this study the projection expressed in the Eq. 2 was used:

$x_{i}=\frac{x_{i}^{0}}{u_{i}}$, where $x_{i}^{0}$-the $\mathrm{i}$-th component of the source dimensional vector, $u_{i}$-the unit of the $\mathrm{i}$-th component of the dimensional vector, $x_{i}$ - the value of the $\mathrm{i}$-th component after the standardization of the decision space.

For the tests being performed, the following were adopted respectively: $\mathrm{u}_{1}=2$ for the Ai angle, $\mathrm{u}_{2}=0.5$ for the feed ratio and $\mathrm{u} 3=10$ for the $\mathrm{Lt}$ length of the horizontal semi-axis of the ellipse limiting the trajectory of movements. Out of the standardized data the most similar case is searched for with the use of the $(\delta)$ Euclidean measure expressed by the Eq. 3 .

$\delta_{n}=\sqrt{\sum_{i=1}^{k}\left(x_{i}-x_{i}^{n}\right)^{2}}$,

where $\mathrm{n}$-index of the case from the database, $\mathrm{k}=3$-number of the dimensions of the decision space.

It is assumed that the smaller the Euclidean distance of the problem is from the case in the database the more similar the problem is to the case. In the CBR method weights are often attributed to individual variable parameters. In this paper all weights were established on a fixed level $=1$, which gives the mentioned example more clarity. Most often during initial examination, the designer has no indications as to which input parameters are preferable.

In Fig. 9 the stability regions are presented, which were determined by the CBR method for the data derived from the results obtained for the minimum plan, central composite one, and the full plan. Considerable differences may be noticed in the evaluation of the solutions for the minimum plan and central composite one, whereas the differences between the central composite plan and the full one are relatively small. However, both the results of the minimal plan as well as those of the central composite one suggest placing the solution that has been verified negatively in the full plan in the stability region $(\mathrm{Ai}=8$, Feed_ratio $=1.5, \mathrm{Lt}=$ 

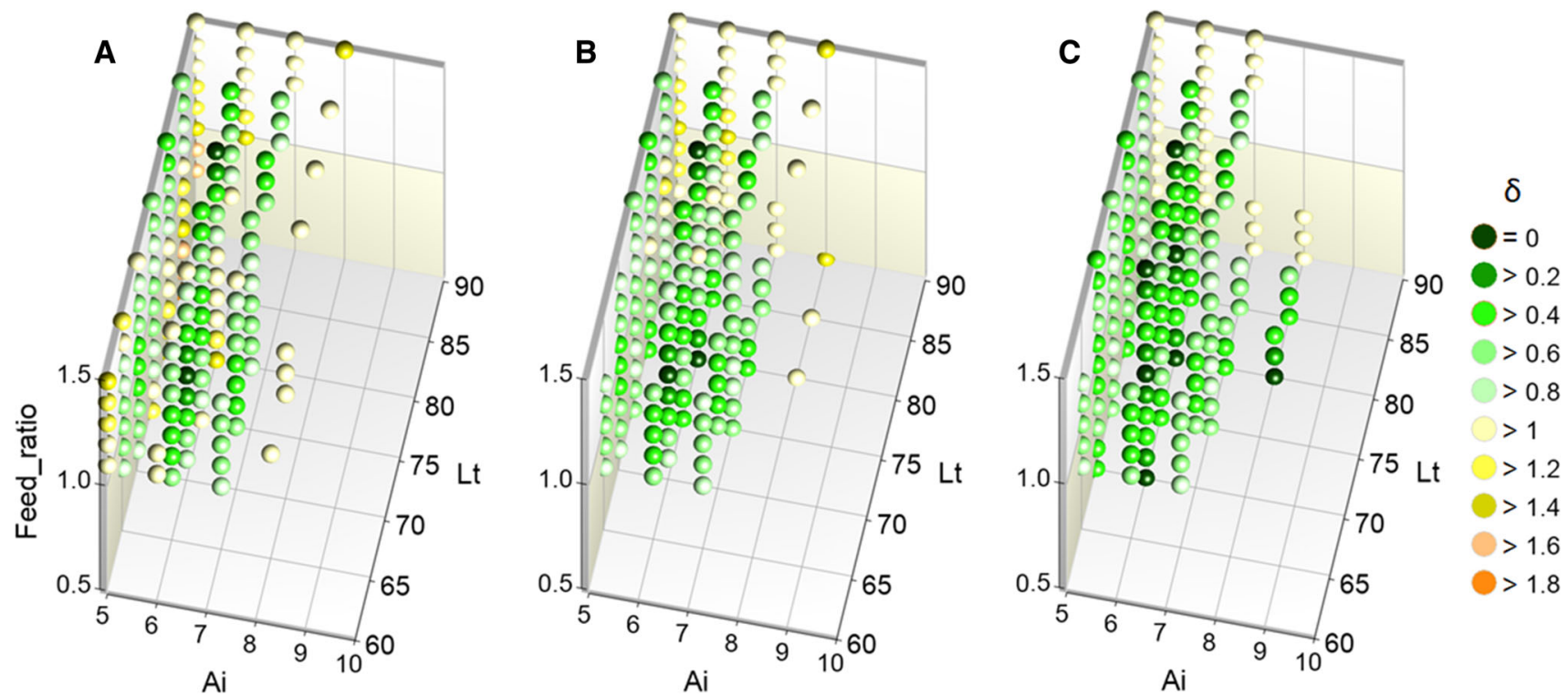

Fig. 9 Distribution of the $\delta$ Euclidean measure for the positions in the stability region: for the minimal plan (a), central composite one (b), and full plan (c)

80). Therefore, one should approach the evaluation of the solutions located on the border of the stability region with reserve.

\section{Classification using the potential function method}

The potential function should meet the following conditions: it has to be positive everywhere and it has to decrease as the distance between the $\delta$ point and the source increases. In the CBR method the $\delta$ value may be assumed to be the Euclidean distance in the standardized decision space. Often, the proposed form of the potential function is the formula (4).

$$
f(\delta)=1 / e^{\left(b \delta^{2}\right)},
$$

where: the " $b$ " coefficient is assumed to be equal to 5 so that the influence of the potential source fades out after exceeding the half of the range of the value of the given variable (Fig. 10) assumed in the experiment, which corresponds to the value of 1 in Euclidean metrics.

One point of the decision space can be located in the area of the influence of a few potential sources belonging to various classes. In the example being analysed the sources should be associated with the examples from the teaching set divided into two classes: "part OK" and "part with defects". The authors have proposed a certain modification to the potential function method. The value of influence of a given source after exceeding the distance of 1 from the source admittedly disappears, but the $D c$ certainty coefficient of the affiliation of the examined case with the "part OK" class attains the value of 0.5 in such instance (Fig. 10). This is in conformity with the heuristics which is difficult to negate, whereby if the examined case is out of the range of the influence of any source then, on this account, the certainty of the affiliation with one of two classes should be the same. Hence, two formulas have been assumed (5) and (6) defining the impact of a given source on the degree of affiliation with the "part OK" class.

$$
\begin{aligned}
& f_{1}(\delta)=0.5+0.5 / e^{\left(b \delta^{2}\right)}, \text { if part OK } \\
& f_{2}(\delta)=0.5-0.5 / e^{\left(b \delta^{2}\right)}, \text { if part with defects }
\end{aligned}
$$

In order to map the affiliation of the analysed point of the decision space with the "part OK" category the influence of all the sources has to be taken into account according to the Eq. 7 where the $\oplus$ operator is understood as a soft logic sum (Eq. 8) if the i-th source belongs to the "part OK" set. In the opposite instance, when the source belongs to the "part with defects" category a soft logic product (Eq. 9) is applied:

$$
\begin{aligned}
D c= & \sum_{i=1}^{n} \oplus f\left(\delta_{i}\right) \\
S_{a}^{\prime}= & 0.5+\left(S_{a}-0.5\right)+\left(f_{1}\left(\delta_{i}\right)-0.5\right) \\
& -\left(S_{a}-0.5\right) \cdot\left(f_{1}\left(\delta_{i}\right)-0.5\right) \\
S_{a}^{\prime}= & 2 S_{a} \cdot f_{2}\left(\delta_{i}\right)
\end{aligned}
$$




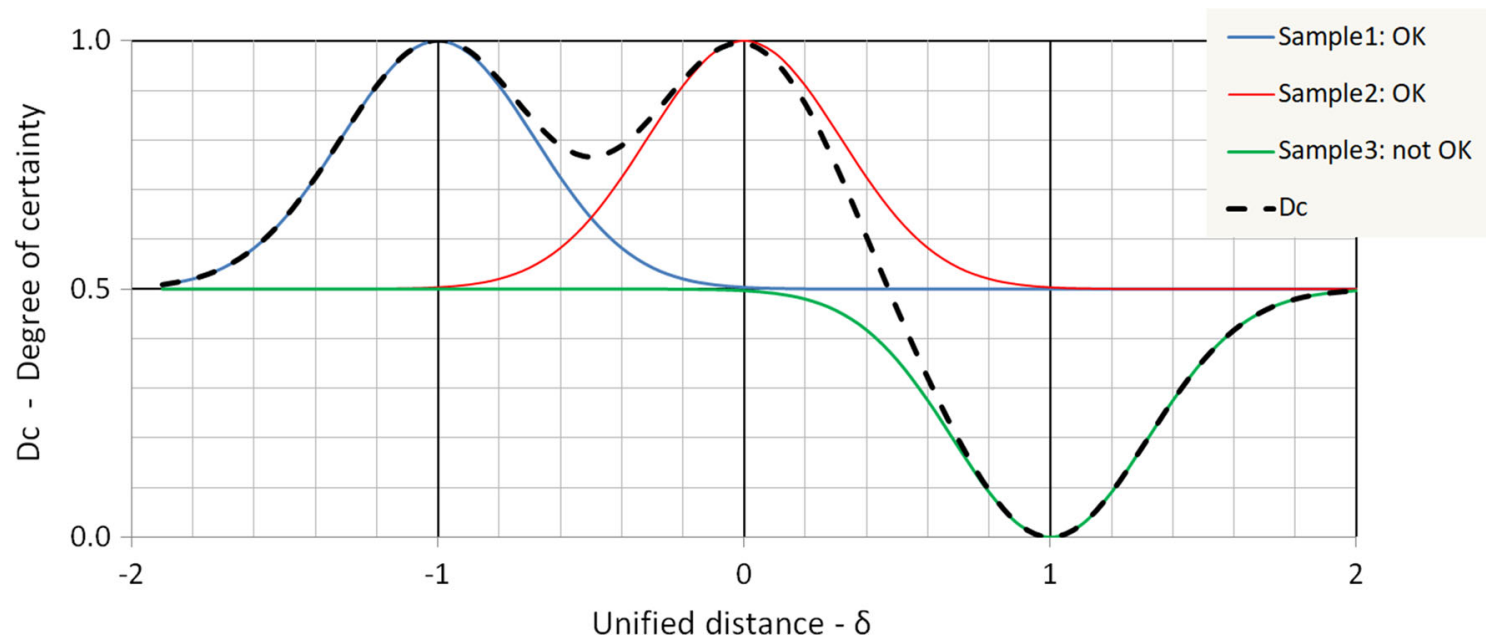

Fig. 10 An example of the formation of the $D c$ degree of certainty with 3 influence sources in the single-dimensional decision space

where $\mathrm{n}$-number of sources (elements of the teaching set), Dc-the degree of affiliation with the "part OK" category, $S_{a}$ - the current value of the sum of influences.

The form of these equations results from the heuristic premises:

- If the point being checked is closer to the successive source of the "part OK" type, then its classification to that class should increase,

- If the point being checked is at the same distance from the representatives of both classes, then its degree of affiliation with the "part OK" class should be 0.5 .

- If the point being checked is outside the influence of any source, then its degree of affiliation with the "part OK" class should also be 0.5 .

The two last premises prove the lack of knowledge on the affiliation with one of the analysed categories. After taking into account the influence of all sources we adopt the $D c$ degree of the affiliation with the "part OK" category as the current $S_{a}$ value. An example of taking into account the influence of 3 sources in single-dimensional decision space by the method referred to above is illustrated in Fig. 10.

In comparison with the CBR method the $D c$ coefficient allows one to take into account the impact of all teaching set elements depending on their distribution in the decision space. Figure 11 presents the stability regions including the area of the decision space for which the $D c$ is higher or equal to 0.5 , said regions determined for the experiments including 9,15 , and 27 trials, respectively. One can observe that the stability region expands along with the increase of the number of trials, and also the $D c$ degree of certainty increases for the stability region core. This testifies to the correctness of the projection of the knowledge acquired in higher number of trials. The potential function method performed for the min- imal plan and the central composite one have been verified positively for all the trials carried out within the full plan, and this revels its advantage over the CBR method.

\section{Classification with the many adaptive linear elements (Madaline) method}

The typical neural networks have one or 2, 3 indirect layers which, in actual fact, introduce a "black box" element into the model. In the proposed case of the model outline inspired by the Madaline method that element does not occur. All the elements of the designed network are intelligible and determined only by the examples constituting the teaching set. This concerns both the network topology as well as all the weights attributed to the curves. In addition, there is no need to teach the network to recognize the objects from the teaching set treated as standards!

The starting point was the observation that two independent observers being in two different points of the space, preferably outside the decision space, and observing two points of that space located close one to another, cannot find independently whether those points are located in close proximity. When the observers referred to above exchange the results of their observations, they are able to answer that question, This situation is illustrated in Fig. 12 where nonparallel unit vectors $V^{1 A}=\left[x_{1}^{1 A}, x_{2}^{1 A}, x_{3}^{1 A}\right]$ and $V^{2 A}=$ $\left[x_{1}^{2 A}, x_{2}^{2 A}, x_{3}^{2 A}\right]$, with the direction determined from the observer's point to the observed point " $\mathrm{A}$ " are connected with the observers' points " $\mathrm{O}_{1}$ " and " $\mathrm{O}_{2}$ ".

Let us assume that the " $\mathrm{A}$ " point is a representative of the trial belonging to the teaching set, the classification result of which (i.e. the trial) \{ "part OK" "part with defect"\} is known. This point is something like a standard and it may be surmised that the points located nearby it belong to the same class. One ought to establish whether the "B" point 

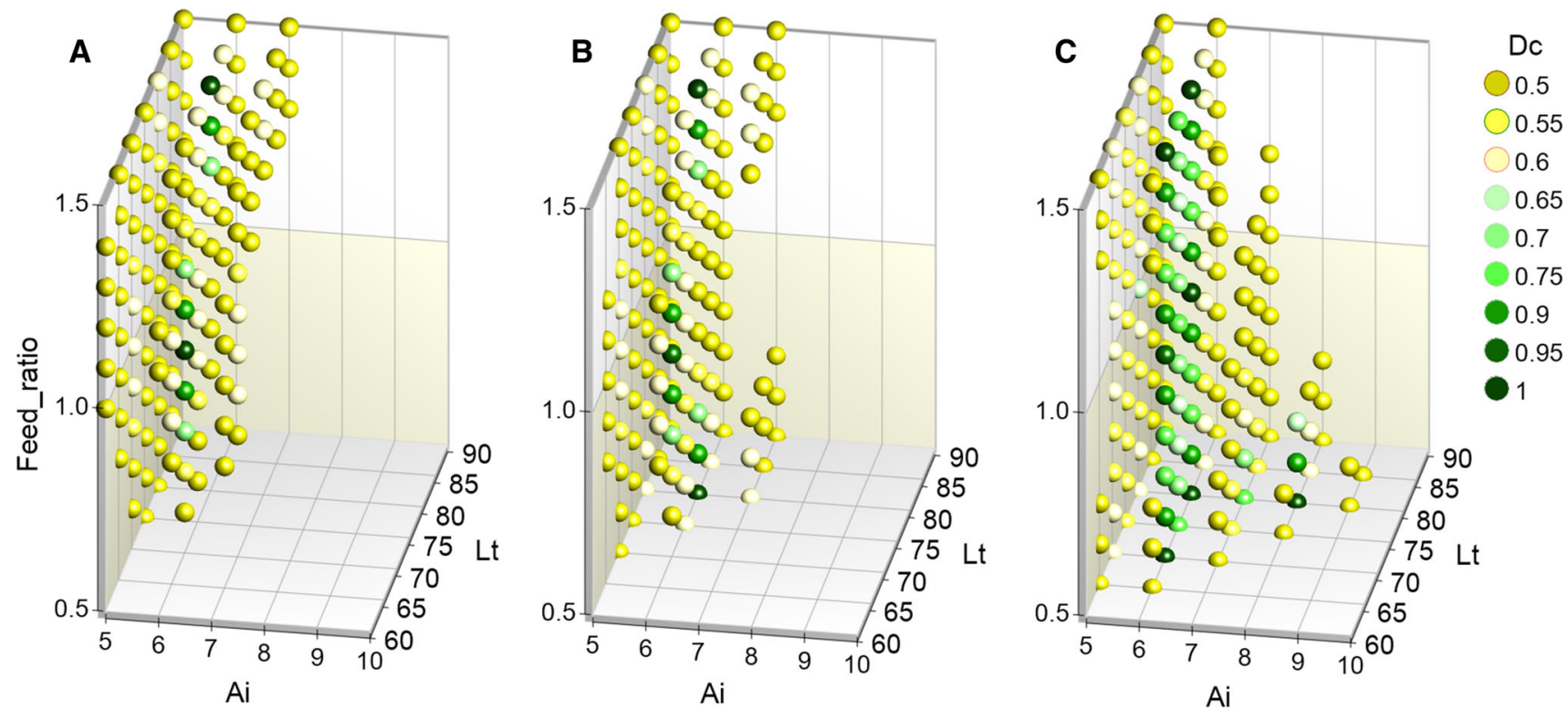

Fig. 11 The stability regions determined by the potential function method for the minimal plan (a), central composite one (b) and the full plan (c)

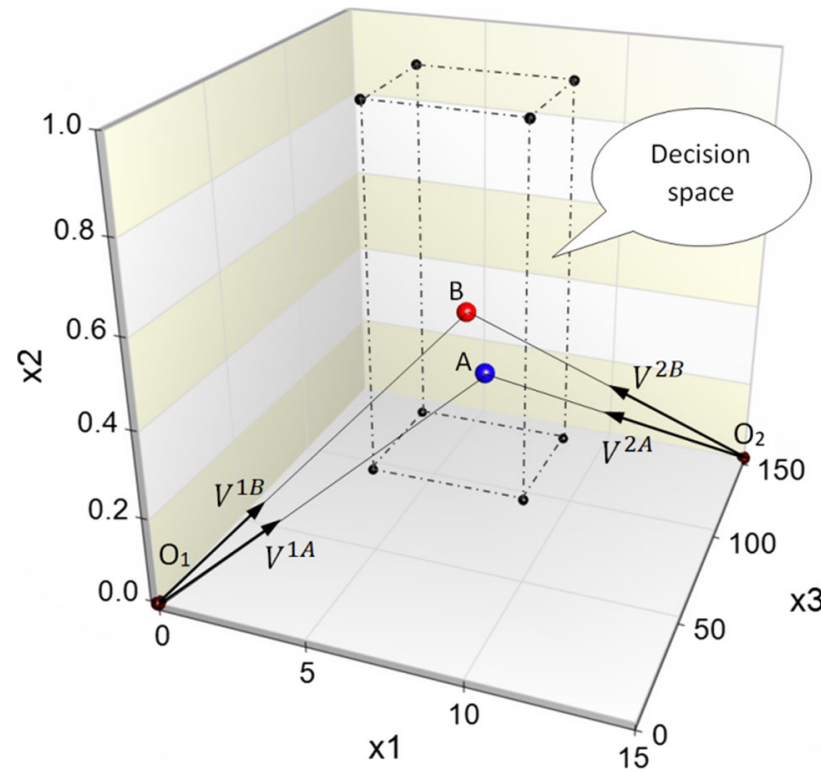

Fig. 12 Correct distribution of $\mathrm{O}_{1}$ and $\mathrm{O}_{2}$ observation points in relation to the situation of the decision space

determined by the unit vectors $V^{1 B}=\left[x_{1}^{1 B}, x_{2}^{1 B}, x_{3}^{1 B}\right] \mathrm{i}$ $V^{2 B}=\left[x_{1}^{2 B}, x_{2}^{2 B}, x_{3}^{2 B}\right]$, is located close to "A". This can be determined based on the simultaneous similarity of $V^{1 A}$ to $V^{1 B}$ vector and $V^{2 A}$ to $V^{2 B}$. Since the vectors referred to above are normalized then in numerical terms their similarity is expressed by the cosine of $\varphi$ angle between the vectors in one pair, and the cosine is calculated as the scalar product according to the formulas (10) and (11):
$Y_{1}=\cos \varphi_{1}=V^{1 A}\left(V^{1 B}\right)^{T}=x_{1}^{1 A} x_{1}^{1 B}+x_{2}^{1 A} x_{2}^{1 B}+x_{3}^{1 A} x_{3}^{1 B}$,

$Y_{2}=\cos \varphi_{2}=V^{2 A}\left(V^{2 B}\right)^{T}=x_{1}^{2 A} x_{1}^{2 B}+x_{2}^{2 A} x_{2}^{2 B}+x_{3}^{2 A} x_{3}^{2 B}$.

The $Y_{1}$ and $Y_{2}$ values can be treated as a subjective evaluation of similarity expressed by the observer 1 and 2 respectively. Therefore, the measure of the objective similarity of $\mathrm{Y}$ can be the sum Y1 + Y2. The technical execution of the calculations referred to above is the linear neural network in the configuration shown in Fig. 13. As it can be seen, the weights of the connections to the indirect layer are immediately the inputs generated for the standard (pattern). Obviously, an effort could be made to teach such a network but with its course being optimal the result should be just like that.

By extending the above structure to all the cases registered in the teaching set we receive the network as in Fig. 14. In the input layer, in the instance of 3-dimensional decision space and two observers, there is always a constant number of six neurons. In the indirect layer the number of neurons is $2 \mathrm{~N}$, where $\mathrm{N}$ corresponds to the numerical strength of the teaching set. In the output layer the number of neurons is $\mathrm{N}$. When providing the data describing the $\mathrm{j}$-th element from the teaching set as the input, we obtain the value $\mathrm{Y}^{\mathrm{j}}=2$ in the output. Other outputs assume values $<2$. For an element from outside of the teaching set, the values are always $<2$, but the maximum $\mathrm{Y}^{\mathrm{m}}$ value obtains the output representing the teaching element most similar to the decision space point which is tested. 


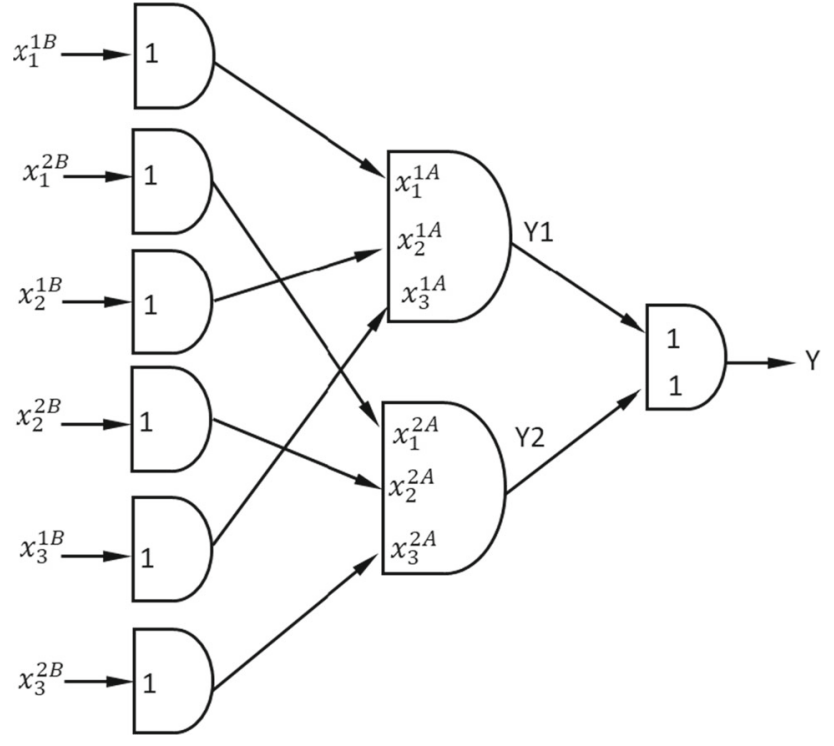

Fig. 13 A fragment of the network recognising I standard (pattern)

The determined stability regions for the minimal plan (A), central composite one (B) and the full teaching set (C) respectively are illustrated in Fig. 15. As demonstrated in that illustration, the stability region for the test with 9 trials is quite extensive, which proves that the approach was too optimistic. The results being more adequate in comparison with reality are projected by the stability region construed for 15 standards (patterns). The stability region generated out for the full plan does not diverge from the RSD, CBR and PFM methods, in terms of quality, for 27 evenly distributed tests.

The Madaline method does not give the measure of the affiliation degree between the decision space point and the stability region. One can only be guided by the principle that the centre of the stability region is characterized by a lower risk that a production defect may occur. The optimal selection of parameters should therefore be dependent on the time of the production cycle. Figure 16 presents the assignment of the corresponding cycle time to the points in the stability region. As it could be expected, the shortest times correspond to high feed speed, therefore the upper plane limiting the stability region. Those limitations result from the selected borders of the decision space. Therefore, the general conclusion is: in this case the search for higher productivity should proceed towards the trials with increased feed speed. The practical tests conducted have confirmed that the area of the stability region can be shifted towards the higher values of feed speed.

\section{Determination of the set of Pareto-optimal solutions}

The multi-criterion optimization consists in finding the undominated solutions which are a compromise from the

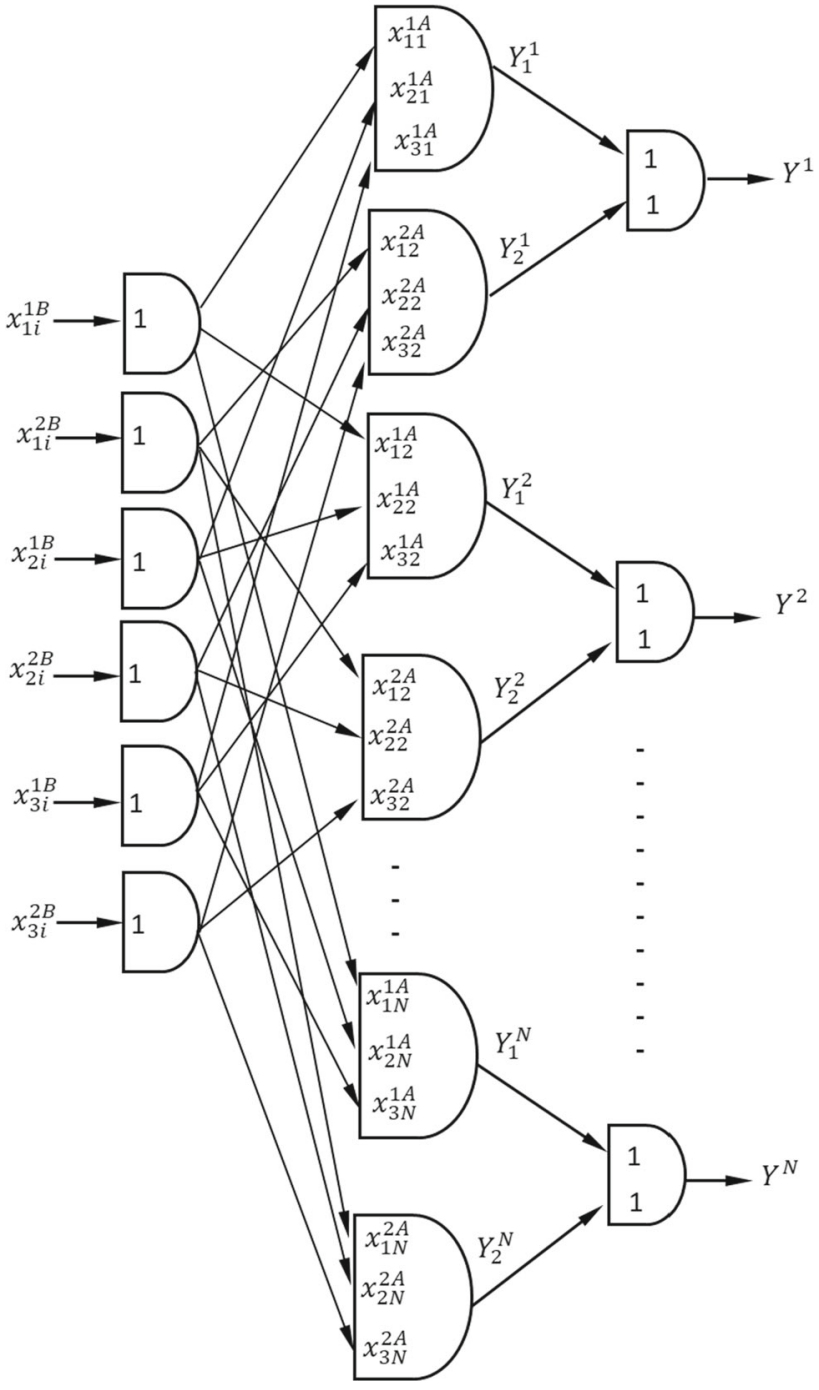

Fig. 14 The topology of the classifier of the points in the decision space

point of view of individual criteria. The issue of the optimal selection of the variable parameters of the spinning process comes down to the two-criterion optimization where the first $F_{1}$ criterion is the minimization of the risk of producing a defective part, and the second $F_{2}$ criterion is the minimization of the spinning cycle time. Three methods out of those proposed in this study are suitable for starting the procedure of the two-criterion optimization. These include the RSD method, where the higher value of response corresponds to the higher certainty of obtaining a correct part, the CBR method where the risk of producing a defective part can be linked with the Euclidean distance from the correct solutions, and the PFM one in which the degree of the affiliation with the set of correct solutions is determined directly.

A 3-dimensional discrete decision space is given where every point of the space is represented by the $X=\left[x_{1}, x_{2}\right.$, $\left.x_{3}\right]^{\mathrm{T}}$ vector. Every $x_{i}$ component of the $\mathrm{X}$ vector has a specific 

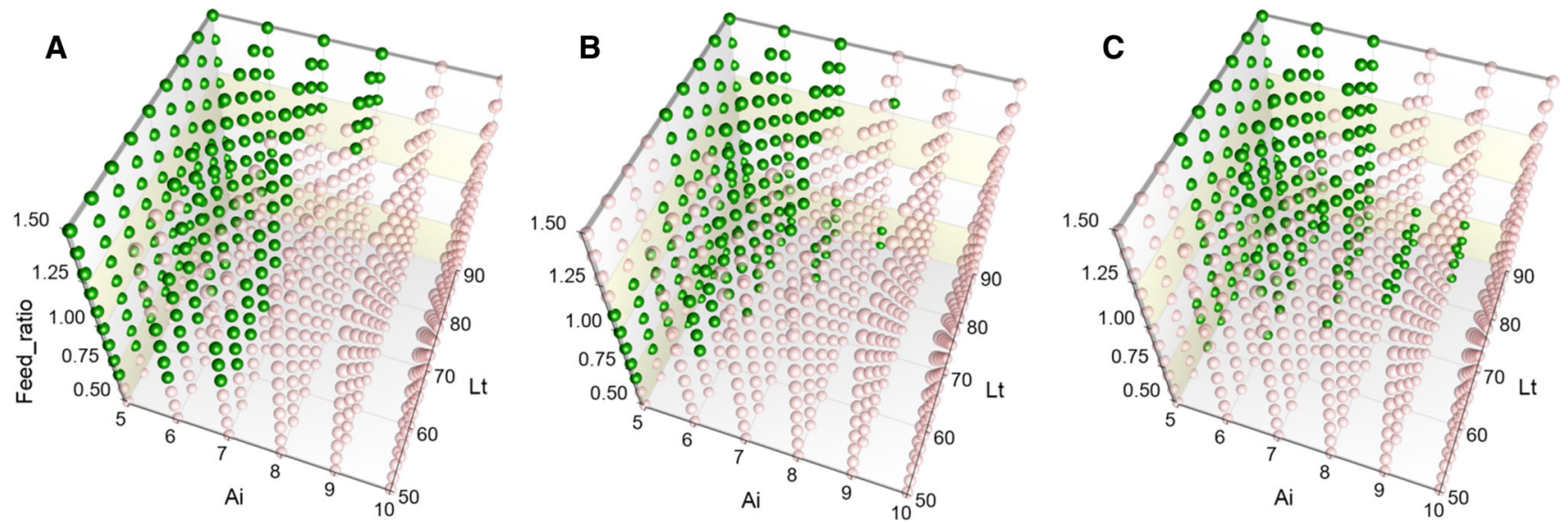

Fig. 15 The stability region (green) determined with the Madaline method for the minimal plan (a), central composite (b) one, and full plan (c) (Color figure online)

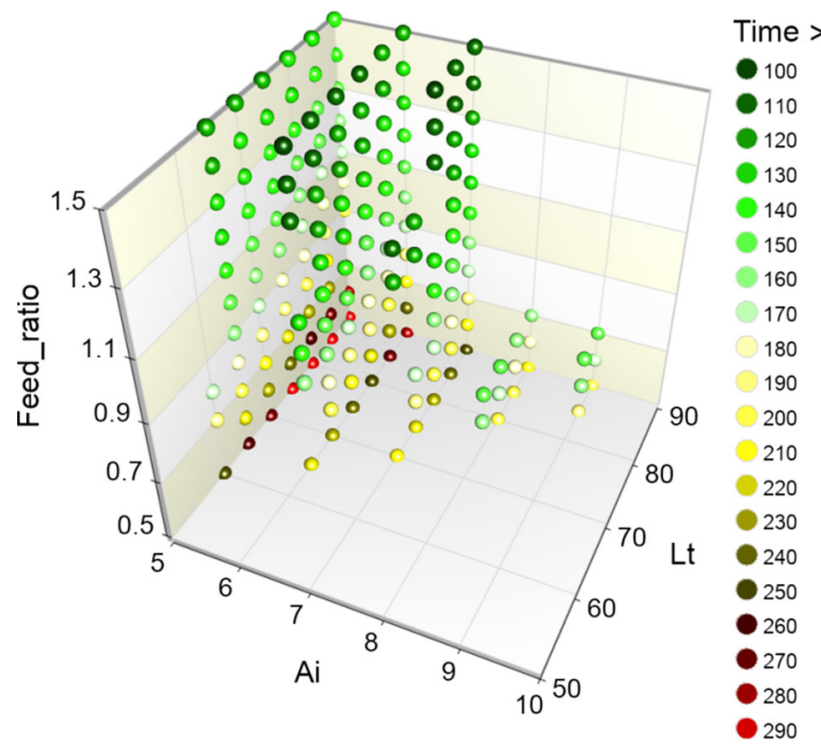

Fig. 16 Distribution of the duration for the stability region obtained from the plan of the full experiment

range of variability and constant variability gradient in that range. Also, a vector of the purpose function: $F(X)=\left[F_{1}(X)\right.$, $\left.F_{2}(X)\right]^{\mathrm{T}}$ has also been formulated. Formally, the problem of two-criterion optimization consists in finding, considering the existing limitations, the set of the $X_{i}$ vectors for which the appropriate components of the $F\left(X_{i}\right)$ purpose function vector meet the conditions formulated in the expressions (12) and (13):

$\forall_{k \neq i}$ iff $F_{1}\left(X_{i}\right)>F_{1}\left(X_{k}\right) \Rightarrow F_{2}\left(X_{k}\right)>F_{2}\left(X_{i}\right)$,

$\forall_{k \neq i}$ iff $F_{2}\left(X_{i}\right)>F_{2}\left(X_{k}\right) \Rightarrow F_{1}\left(X_{k}\right)>F_{1}\left(X_{i}\right)$.

The expressions (12) and (13) correspond to the situation when one strives for the minimization of both criteria. This takes place in the case of the CBR method where the similarity was determined by the distance in the Euclidean metrics. For the PFM and RSD methods we maximize the degree of affiliation or the response plane, therefore the expressions (12) and (13) should be replaced with the expressions (14) and (15):

$\forall_{k \neq i}$ if $F_{1}\left(X_{i}\right)\left\langle F_{1}\left(X_{k}\right) \Rightarrow F_{2}\left(X_{k}\right)\right\rangle F_{2}\left(X_{i}\right)$

$\forall_{k \neq i}$ if $F_{2}\left(X_{i}\right)>F_{2}\left(X_{k}\right) \Rightarrow F_{1}\left(X_{k}\right)<F_{1}\left(X_{i}\right)$

In Figs. 17, 18, 19, 20, 21 and 22 the Pareto sets for the RSD, CBR and PFM methods are presented, respectively, as well as the Pareto front. The obtained results differ slightly which means that a lower number of experiments, and thus a smaller number of production rejections and shorter time does not have to mean that a substantially worse solution was obtained. The fact that all the Pareto-optimal solutions have been obtained for the maximum tested feed $=1.5 \mathrm{~mm} / \mathrm{rev}$. indicates clearly that the desired shift of the Pareto front towards decreasing the cycle time can be achieved by increasing the feed speed. The most numerous Pareto set has been generated in the RSD method. A large number of the solutions offered thereby is characterised by long cycle times with a small increase of $\hat{Y}$. For this reason, being guided by the shape of the Pareto front is a prudent solution.

There are a few aspects in the evaluation of the quality of the set of Pareto-optimal solutions, i.e. cohesion as the minimum distance between adjacent solutions on the Pareto front, diversity as their even distribution in the decision space, and the maximum range of the Pareto front in the space of criteria. The obtained sets of the Pareto-optimal solutions are not numerous, what results from the number 594 of the points being analysed in the discrete decision space. The number of the points being analysed is determined by the assumed ranges and the gradients of the variability of the parameters 

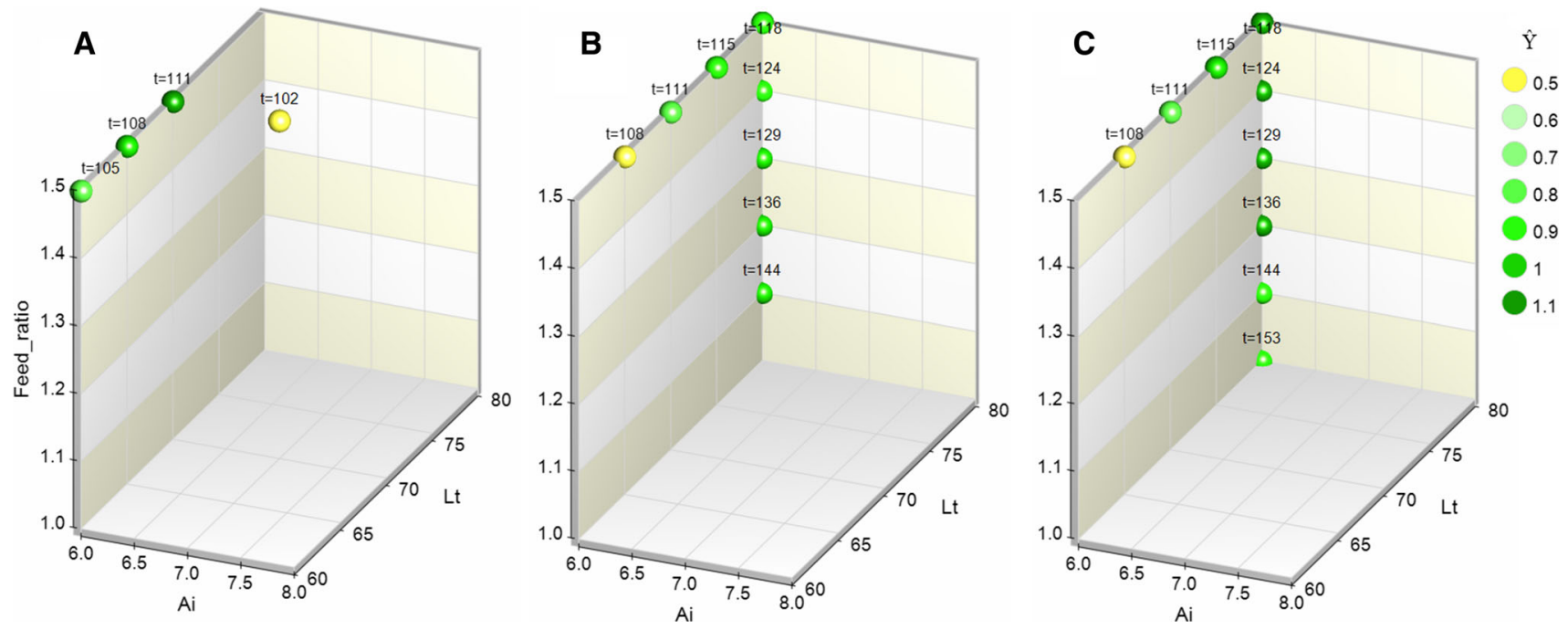

Fig. 17 Pareto set for the RSD method for the plans: minimal (a), central composite (b), and full (c)
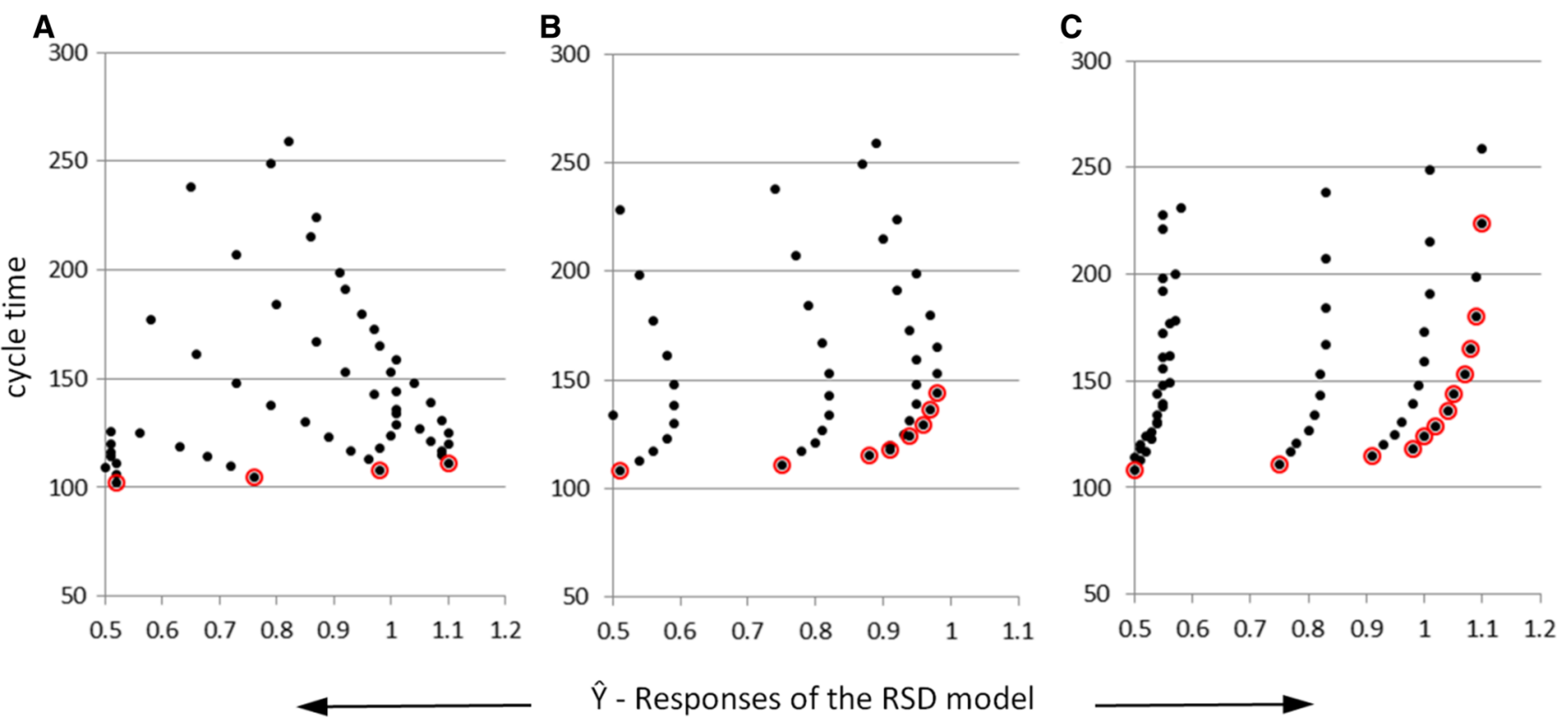

Fig. 18 Pareto front for the RSD method for the plans: minimal (a), central composite (b), and full (c)

defining the 3-D decision space. The feed gradient was established as $0.1 \mathrm{~mm} / \mathrm{rev}$., the $A_{i}$ angle as $1^{0}$, and the length of the horizontal $L t$ semi-axis of ellipse as $5 \mathrm{~mm}$. In order to obtain a better quality of the Pareto front; the number of the considered decision space points should be definitely increased and smaller gradients for individual variable input parameters should be introduced. For instance, the assumption of the variability gradients $A_{i}=0.1^{\circ}$, Feed_ratio $=0.01$ and $L t=1$ would increase the number of the points under consideration to over 200,000 . In such a situation some problems constitute a second criterion since in practice we can determine the cycle time through a simulation for several hundred points only-for the other ones the cycle time has to be approximated.

With such high number of points being considered more sophisticated methods for the determination of the Pareto set should be used. Since it has been assumed that this paper refers to simple heuristic methods only, the description of those methods has been omitted. At the same time, we encourage the readers interested in this subject to familiarize themselves with the available literature in this field. In the study (Stryczek and Pytlak 2014) a modified method of multiparticle optimization (PSO) was proposed for the issues of multi-criterion optimization with the discrete decision space. In the PSO method the way of the determination 

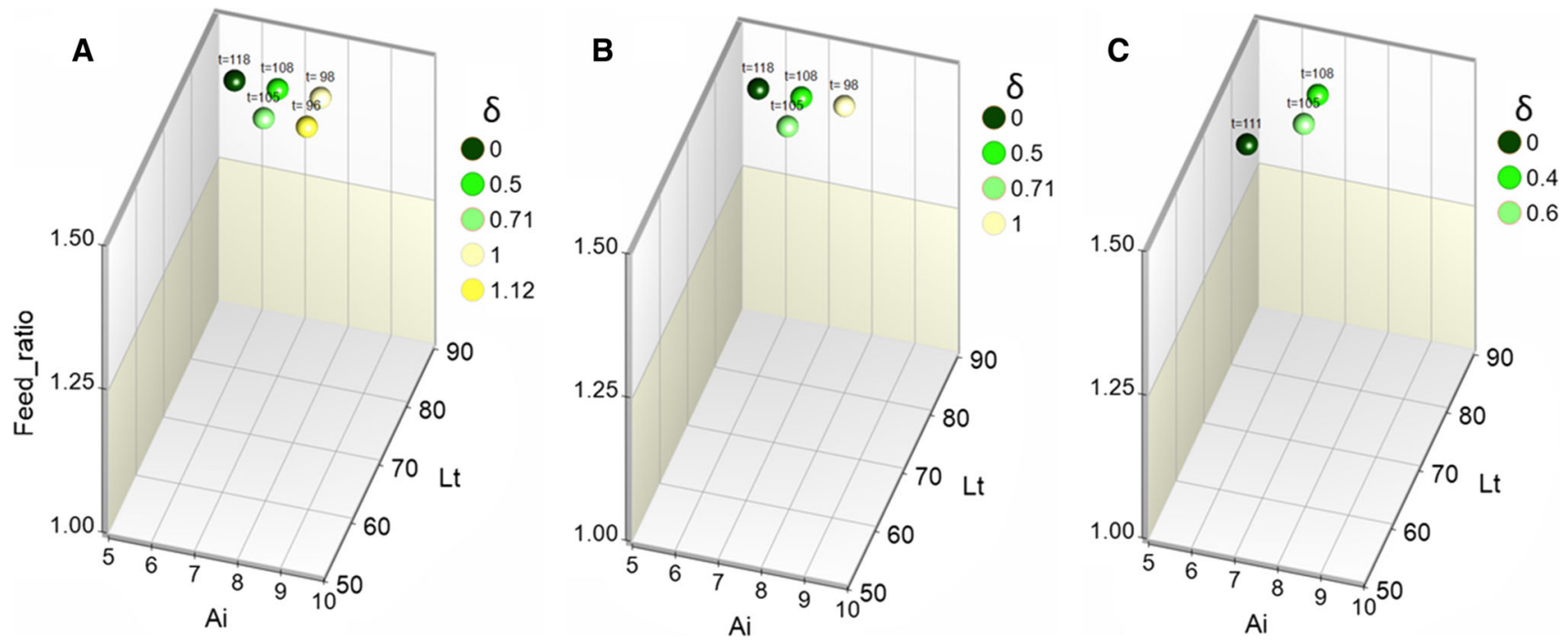

Fig. 19 Pareto set for the CBR method for the plans: minimal (a), central composite (b), and full (c)
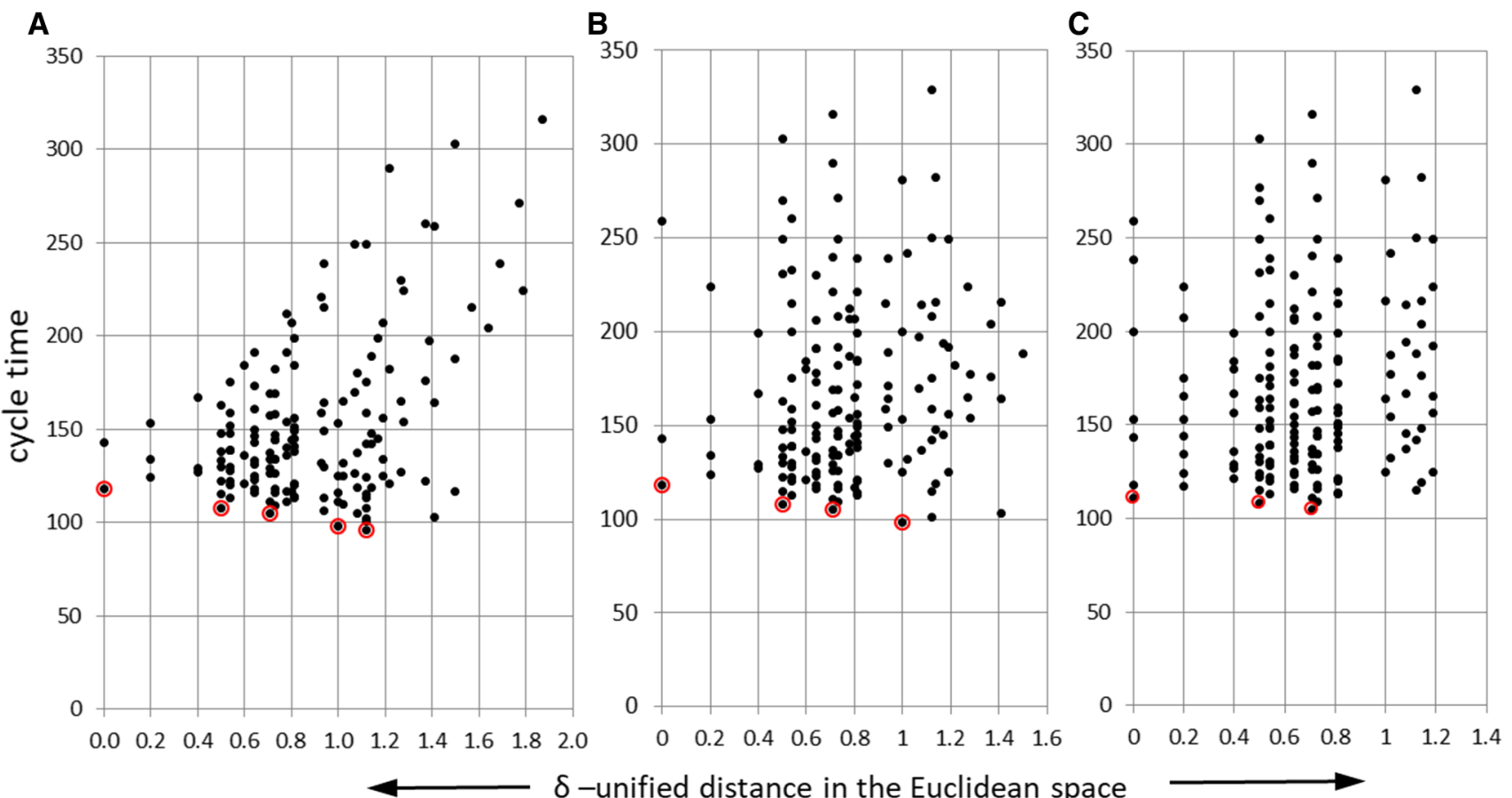

Fig. 20 Pareto front for the CBR method for the plans: minimal (a), central composite (b), and full (c)

of the moment of inertia, teaching coefficient, and social coefficient was changed. In addition, the elitism and an innovative mechanism of braking particles protecting them from exceeding permissible limits of the decision space were introduced. A faster similarity (coincidence) of the PSO method in comparison with the genetic algorithms was confirmed. To evaluate the quality of the generated Pareto set, the assessment based on the measurement of entropy and quality index (IDG) were determined and compared.

\section{Summary}

The attention of the authors of the presented study is focused on the comparative analysis of the practically useful techniques for the modelling of machine spinning process. The term "practically useful" should be interpreted as easy for unassisted development by the direct user and quick in the generation of solutions. In fact, for all the presented modelling methods appropriate software can be provided as functional macros in the commonly available and well known 

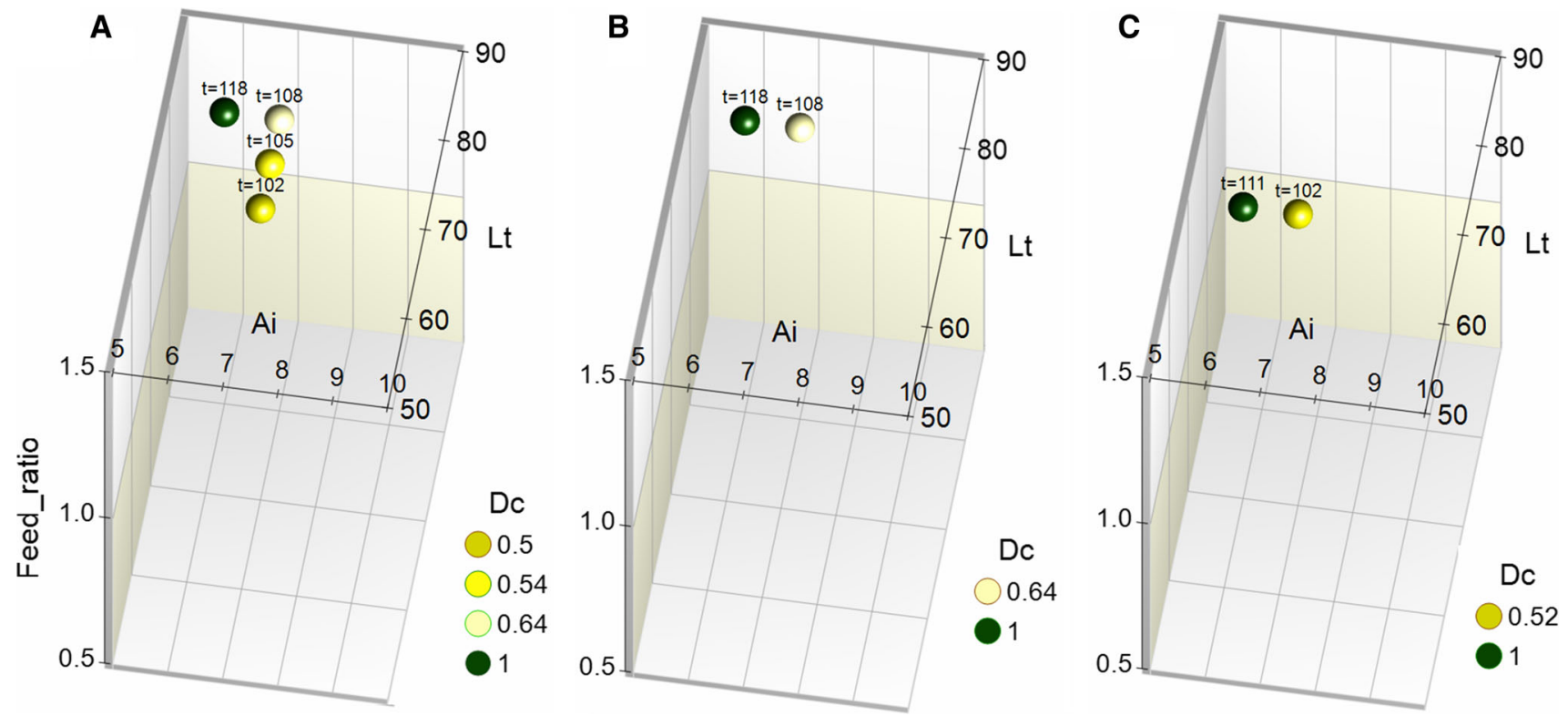

Fig. 21 Pareto set for the PFM method for the plans: minimal (a), central composite (b), and full (c)

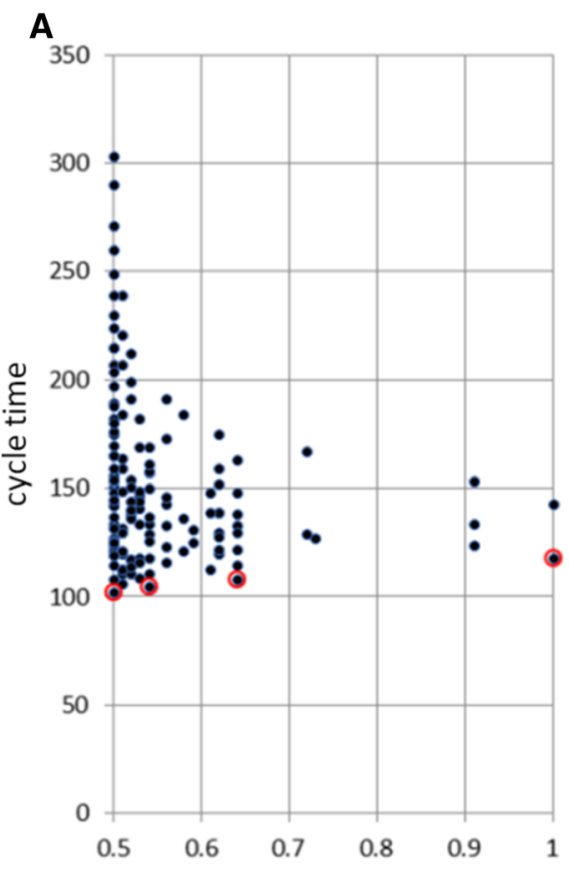

B

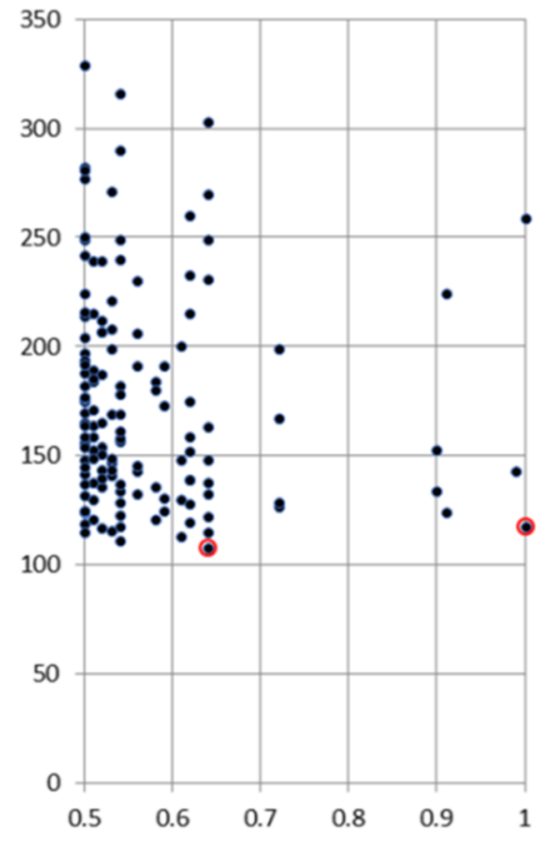

C

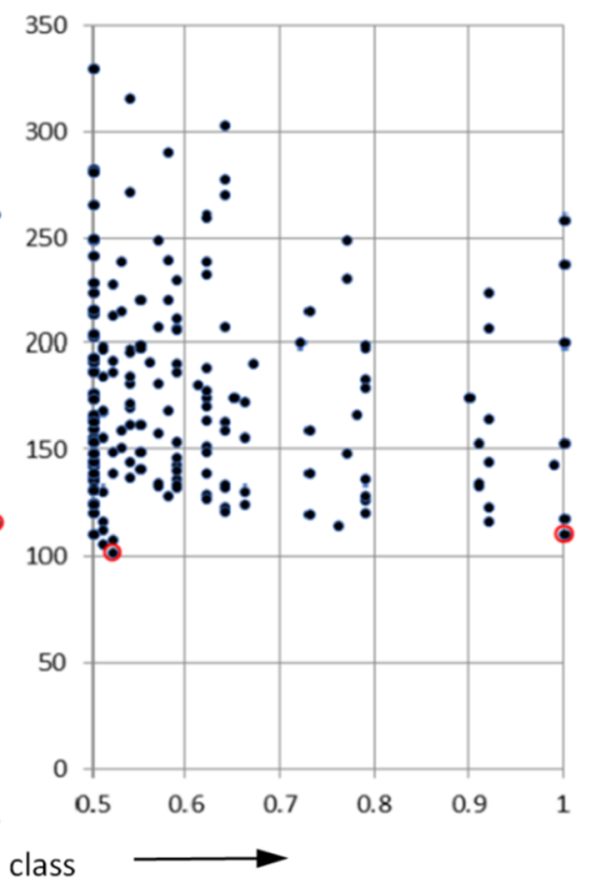

Fig. 22 Pareto front for the PFM method for the plans: minimal (a), central composite (b), and full (c)

to engineers calculation sheet formats. They are practically useful also because in spite of their relative simplicity they are capable of remembering the user's knowledge without application of expert systems having and advanced form. Therefore, these methods can be classified as belonging to the categories of intelligent methods since basing on incomplete information and uncertain knowledge they can suggest some satisfactory solutions to the user.
When evaluating the proposed approaches it should be stated that:

- The CBR method without taking into account the Euclidean measure is not commendable since it generates solutions charged with high risk of failure as correct ones. The application of this method may be thoroughly justified 
provided that the user will be searching for the solutions in the central parts of the stability region.

- The CBR method supported by the evaluation of the Euclidean measure can suggest faulty solutions. However, it seems to be a good compromise between the calculation complexity and the adequacy of the proposed solutions, provided that the solutions from the stability region border are avoided.

- The potential function method suggests a credible form of the stability region already for a small number of trials in experimental tests. This allows for skipping the full plan and thereby limit the production rejections at the initial production stage. Despite a higher degree of calculation complexity this method is commendable.

- Only the PFM and RSD methods take into account the distribution of good and bad solutions. Thus, they use the information contained in the set of known solutions to the full extent.

- Determination of the stability region facilitates making decisions for the user with regard to the direction of further research but a more pertinent information is supplied by the Pareto set distribution. It should be noted that the Pareto-optimal solutions coincide in the CBR and PFM method, regardless of the number of the tests carried out, and this should prefer the minimal plan of an experiment.

- If the elements belonging to the Pareto set are located on the edge of the stability region then it is obvious that in successive trials performed as a passive experiment one should go outside this region if only no technical contraindications occur.

- The Madaline method is worthy of attention but only in the situation when a vast and representative teaching set is available, which is a characteristic feature of the most applications of artificial neural networks.

- In the CBR and PFM method including a successive case causes no problems. In the RSD method repeating model generation is required. In the proposed Madeline method variant, a change of the network structure is required, and because of that it should be ranked the lowest.

- The classification of the points situated slightly outside of the assumed decision space was also analysed. The best results were obtained for MFP, and definitely the worst for RSD, where they significantly diverged from the assumed range $[0,1]$ and were very difficult to interpret.

The method covered in this study, based on the Madaline network model with a priori attributed weights is an original idea of the authors. The authors do not know any examples of such an approach to the construction of the model. At the same time the authors are aware that the proposed approach questions many scientific studies using the networks of Madaline type, whose authors made-maybe unnecessarily_an effort to establish the weights through teaching the network. The authors hope that this controversy will open a scientific discussion on this subject.

Open Access This article is licensed under a Creative Commons Attribution 4.0 International License, which permits use, sharing, adaptation, distribution and reproduction in any medium or format, as long as you give appropriate credit to the original author(s) and the source, provide a link to the Creative Commons licence, and indicate if changes were made. The images or other third party material in this article are included in the article's Creative Commons licence, unless indicated otherwise in a credit line to the material. If material is not included in the article's Creative Commons licence and your intended use is not permitted by statutory regulation or exceeds the permitted use, you will need to obtain permission directly from the copyright holder. To view a copy of this licence, visit http://creativecomm ons.org/licenses/by/4.0/.

\section{References}

Aizerman, M., Braverman, E. M., \& Rozonoer, L. I. (1964). Theoretical foundations of the potential function method in pattern recognition learning. Automation and Remote Control, 25, 821-837.

Auer, C., Erdbrügge, M., \& Ewers, R. (2004). Comparison of multivariate methods for robust parameter design in sheet metal spinning. Applied Stochastic Models in Business and Industry, 20, 201-218.

Ewers, R. (2005). Process design and optimisation in sheet metal spinning, Technische Universität Dortmund (in German).

Göbel, R., Kleiner, M., \& Henkenjohann, N. (2005). New approach for process planning and optimization in sheet metal spinning. Advanced Materials Research, 6-8, 493-500.

Gok, A. (2013). Cutting parameter and tool path style effects on cutting force and tool deflection in machining of convex and concave inclined surfaces. International Journal of Advanced Manufacturing Technology, 69, 1063-1078.

Gok, A. (2015a). A new approach to minimization of the surface roughness and cutting force via fuzzy TOPSIS, multi-objective grey design and RSA. Measurement, 70, 100-109.

Gok, K. (2015b). Development of three-dimensional finite element model to calculate the turning processing parameters in turning operations. Measurement, 75, 57-68.

Gok, K., Sari, H., Gok, A., Neseli, A., Turkes, E., \& Yaldiz, S. (2017). Three-dimensional finite element modeling of effect on the cutting forces of rake angle and approach angle in milling. $J$ Process Mechanical Engineering, 231(2), 83-88.

Hayama, M., \& Murota, T. (1963). On the study of metal spinning. Bulletin of the Faculty of Engineering, Yokohama National Univ., $12,53-88$.

Khosravani, M. R., \& Nasiri, S. (2020). Injection molding manufacturing process: Review of case-based reasoning applications. Journal of Intelligent Manufacturing, 31, 847-864. https://doi.org/10.100 7/s10845-019-01481-0.

Kleiner, M., Göbel, R., Kantz, H., Klimmek, Ch., \& Homberg, W. (2002). Combined methods for the prediction of dynamic instabilities in sheet metal spinning. CIRP Annals, 51(1), 209-214.

Li, X., Zhang, S., Huang, R., Huang, B., Xu, C., \& Zhang, Y. (2018). A survey of knowledge representation methods and applications in machining process planning. The International Journal of Advanced Manufacturing Technology, 98, 3041-3059.

Ma, H., Liu, W., et al. (2020). An effective and automatic approach for parameters optimization of complex end milling process based on virtual machining. Journal of Intelligent Manufacturing, 31, 967-984. https://doi.org/10.1007/s10845-019-01489-6. 
Mao, J., Wang, L., Xu, X., \& Newman, S. (2010). A static review of computer-aided process planning research. In Proceedings of the ASME 2010 international manufacturing science and engineering conferences USA. $10.1115 / \mathrm{msec} 2010-34022$.

Mukherjee, I., \& Kumar, Ray P. (2006). A review of optimization techniques in metal cutting processes. Computer and Industrial Engineering, 50(1-2), 15-34.

Music, O., Allwood, J. M., \& Kawai, K. (2010). A review of the mechanics of metal spinning. Journal of Materials Processing Technology, 210(1), 3-23. https://doi.org/10.1016/j.jmatprotec.2 009.08.021.

Rentsch, B. (2018). Virtual design and adaptive control of metal spinning processes. Dissertation, ETH Zurich.

Slater, R. A. C. (1979). A review of analytical and experimental investigations of the spin-forging of sheet metal cones. In $1 s t$ international conference on rotary metal-working processes, $U K$ (pp. 33-60).

Stryczek, R. (2007). Computational intelligence in computer aided process planning: A review. Advances in Manufacturing Science and Technology, 31(4), 77-90.

Stryczek, R., \& Pytlak, B. (2014). Multi-objective optimization with adjusted PSO method on example of cutting process of hardened $18 \mathrm{CrMo} 4$ steel. Eksploatacja i Niezawodnosc Maintenance and Reliability, 16(2), 236-245.

Stryczek, R., \& Wyrobek, K. (2017). Compensation of the trajectory of the shaping roller with complex surface-profile in the machine spinning process. Advances in Manufacturing Science and Technology, 41(3), 5-16. https://doi.org/10.2478/amst-2017-0013.

Vankata Rao, R. (2011). Advanced modeling and optimization of manufacturing process. Springer Series in Advanced Manufacturing, 1, 1. https://doi.org/10.1007/978-0-85729-015-1.

Vergara-Villegas, O., Ramirez-Espinoza, C. F., Cruz-Sánchez, V. G., Nandayapa, M., \& Neco-Caberta, R. (2018). A methodology for optimizing the parameters in a process of machining a workpiece using multi-objective particle swarm optimization. New Perspectives on Applied Industrial Tools and Techniques (pp. 129-151).
Wang, L. (2012). Analysis of material deformation and wrinkling failure in conventional metal spinning process. Dissertation, Durham University.

Widrow, B., \& Lehr, M. A. (1990). 30 years of adaptive neural networks: Perceptron, Madaline, and backpropagation. Proceedings of the IEEE, 78(9), 1415-1442. https://doi.org/10.1109/5.58323.

Ye, Y., Hu, T., Yang, Y., Zhu, W., \& Zhang, C. (2020). A knowledge based intelligent process planning method for controller of computer numerical control machine tools. Journal of Intelligent Manufacturing, 31, 1751-1767. https://doi.org/10.1107/s10845018-1401-3.

Publisher's Note Springer Nature remains neutral with regard to jurisdictional claims in published maps and institutional affiliations. 